\title{
Efectos derivados de la exposición laboral en las mujeres trabajadoras embarazadas expuestas a sustancias peligrosas: revisión sistemática
}

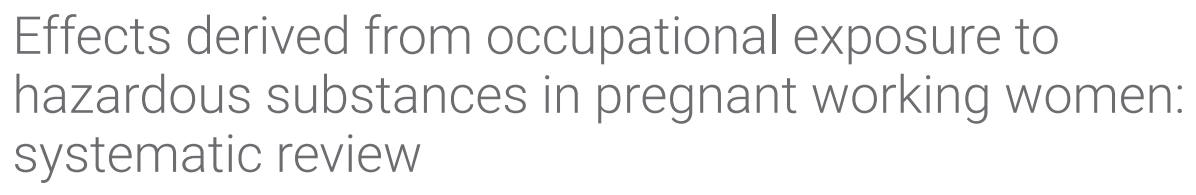

Pablo Barriocanal-Gómez ${ }^{1}$

Carmen $\mathrm{M}^{\mathrm{a}}$ del Pozo-Díez ${ }^{2}$

Olga Kudryavtseva ${ }^{1}$

Iratxe Portillo Chicano² (1) 0000-0001-7758-8349

Javier Sanz-Valero ${ }^{3}$ () 0000-0002-8735-0075

${ }^{1}$ Hospital Universitario de Basurto, Bilbao, España.

${ }^{2}$ Hospital Universitario de Araba, Vitoria, España.

${ }^{3}$ Escuela Nacional de Medicina del Trabajo, Instituto de Salud Carlos III, Madrid, España.

Fechas · Dates

Recibido: 2021.05.26

Aceptado: 2021.07.06

Publicado: 2021.07.15
Correspondencia $\cdot$ Corresponding Author

Javier Sanz-Valero

Correo electrónico: fj.sanz@isciii.es 


\section{Resumen}

Objetivo: Revisar y analizar los efectos derivados de la exposición laboral en las mujeres trabajadoras embarazadas expuestas a sustancias peligrosas.

Métodos: Análisis crítico de los trabajos recuperados mediante revisión sistemática en MEDLINE (PubMed), EMBASE, Cochrane Library, Scopus, Web of Science, LILACS y MEDES, hasta enero 2021. La ecuación de búsqueda se formuló mediante los descriptores «Pregnancy», «Hazardous Substances» y «Occupational Exposure», utilizando también los Entry Terms relacionados y los filtros: «Humans» y «Adult: 19+ years». La calidad de los artículos se evaluó mediante el cuestionario STROBE y el nivel de evidencia y grado de recomendación mediante los criterios SIGN.

Resultados: De las 420 referencias recuperadas (366 de forma digital y 54 manual), tras aplicar los criterios de inclusión y exclusión, se seleccionaron 35 artículos. La evaluación mediante STROBE dio una mediana de 15,32 y según los criterios SIGN se obtuvo un grado de evidencia $2+$ y recomendación C. La obsolescencia de las publicaciones fue elevada (semiperiodo de Burton-Kebler: 22,00; índice de Price: 0\%). En 25 de los 35 estudios revisados la exposición fue a los disolventes orgánicos. En 22 artículos se notificó la aparición de alteraciones congénitas.

Conclusiones: Los trabajos revisados presentaron un alto índice de obsolescencia y un grado de evidencia y recomendación que no permitió asegurar por completo la validez y fiabilidad de las observaciones realizadas. Los resultados mostraron la asociación entre la exposición a sustancias peligrosas con la aparición de diferentes daños para la salud de la trabajadora embarazada y su descendencia. La mayor exposición fue a los disolventes orgánicos y el efecto más observado las alteraciones congénitas.

Palabras clave: Embarazo; Sustancias Peligrosas; Exposición Profesional; Grupos de Riesgo; Solventes; Contaminación Química; Anomalías Congénitas.

\section{Abstract}

Objective: To systematically review and analyze the health effects derived from occupational exposure to hazardous substances in pregnant working women.

Methods: Critical analysis of studies retrieved by systematic review of MEDLINE (PubMed), EMBASE, Cochrane Library, Scopus, Web of Science, LILACS and MEDES, through January 2021. The search strategy was developed by means of the descriptors "pregnancy», «hazardous substances» and "occupational exposure», as well as by using related entry terms and the filters «humans» and «adult: 19+ years». Study quality was assessed using the STROBE questionnaire, and the level of evidence and grade of recommendation via the SIGN criteria.

Results: Out of 420 references identified (366 digitally and 54 manually) and after applying the inclusion and exclusion criteria, 35 articles were selected. The STROBE evaluation yielded a median score of 15.32, and the SIGN criteria a $2+$ level of evidence and a $\mathrm{C}$ grade of recommendation. Article obsolescence was high (Burton-Kebler half-life: 22.0; Price index: $0 \%$ ). In 25 out of 35 studies, the exposure evaluated was to organic solvents. In 22 articles, congenital abnormalities were identified as a health effect. 
Conclusions: This systematic review revealed a high level of obsolescence and a degree of evidence and recommendation that limit the validity and reliability of the observations. Results indicated an association between exposure to hazardous substances and the occurrence of different adverse health effects in pregnant workers and their offspring. The most common exposure was to organic solvents and the most common observed effect were congenital abnormalities.

Keywords: pregnancy; hazardous substances; occupational exposure; risk groups; solvents; chemical pollution; congenital anomalies.

\section{Introducción}

La protección de la maternidad es una manifestación específica en favor de la procreación que, a su vez, forma parte de la protección de los trabajadores especialmente sensibles. La reproducción es un proceso vulnerable, que requiere un equilibrio anatomo-fisiológico, y robusto, del que dan fe la proporción de embarazos y nacimientos normales que se producen en España. Este equilibrio, puede verse alterado por múltiples factores laborales y extralaborales pertenecientes a las esferas cultural, social e individual. Entre los laborales, es preciso señalar, por ejemplo, la utilización o presencia de agentes cada vez más potentes en los procesos industriales (en la lógica búsqueda de unos mayores rendimientos), pero de cuya acción directa o indirecta sobre el trabajador se tiene, muchas veces, nula o escasa información ${ }^{(1)}$.

Como señalaban Rodríguez Ortiz de Salazar et al. ${ }^{(2)}$, en su trabajo de 2005, la protección de la mujer embarazada trabajadora en el ámbito comunitario se reguló por la directiva del Consejo de Europa 92/85/CEE ${ }^{(3)}$, relativa a la aplicación de medidas para promover la mejora de la salud y seguridad en el trabajo de la trabajadora embarazada o en periodo de lactancia. La transposición a la legislación española de la normativa europea se plasmó en la Ley 39/99(4), para promover la conciliación entre la vida familiar y laboral de las personas trabajadoras. Esta Ley, introdujo modificaciones en el Real Decreto Legislativo 1/1994(5), por el que se aprobaba el texto refundido de la Ley General de la Seguridad Social, que en su artículo 38 creaba la nueva prestación de riesgo durante el embarazo, con la finalidad de proteger la salud de la mujer trabajadora embarazada o en periodo de lactancia. La ley 39/99, modificó el artículo 26 de la Ley 31/95(6), de prevención de riesgos laborales, previendo que en los supuestos de maternidad en los que por motivos de salud de la madre o el feto se hiciera necesario un cambio de puesto de trabajo y este cambio no sea posible, se declarará a la trabajadora en situación de riesgo durante el embarazo.

Determinadas condiciones de un puesto de trabajo pueden afectar en la salud de la trabajadora embarazada y en el desarrollo del feto. Por ello, es imprescindible incluirlo en el Plan de Prevención de Riesgos Laborales de la empresa. Elaborar una evaluación de riesgos laborales y tomar las medidas preventivas pertinentes 
es esencial para la promoción de la salud y para la protección de la maternidad en el ámbito laboral(1).

La aplicación de las normas y procedimientos permiten con frecuencia garantizar la protección de la salud y la seguridad de las embarazadas. Pero, algunos de los peligros que existen en el lugar de trabajo pueden afectar a la salud y la seguridad de las mujeres embarazadas o que han dado a luz recientemente y la de sus hijos. Hay que tener en cuenta, que muchas mujeres trabajan durante el embarazo y, otras muchas, reanudan su actividad profesional durante el período de lactancia ${ }^{(7)}$. Así, el estudio de Villar et al.(8), mostró que, en la cohorte estudiada, la mayoría de las trabajadoras mantenían su actividad laboral durante su embarazo, y solo a partir del tercer trimestre se incrementaban las ausencias, sobre todo por incapacidad temporal por contingencia común. En todo caso, estas ausencias del trabajo mostraban una relación consistente con la exposición a riesgos laborales.

En concreto, las sustancias prohibidas para embarazadas y lactantes son aquellas incluidas en la «lista no exhaustiva de agentes y condiciones de trabajo a los cuales no podrá haber riesgo de exposición» por parte de estas trabajadoras establecida por el Real Decreto 298/2009(9), con la finalidad de promover la mejora de la seguridad y de la salud en el trabajo de las trabajadoras embarazadas o en período de lactancia natural.

Por tanto, es necesario conocer la evidencia existente para poder promover acciones preventivas en defensa de la mujer trabajadora embarazada ya que las inadecuadas condiciones laborales se han asociado con una mayor ausencia del trabajo en estas mujeres ${ }^{(10,11)}$ y con un menor autocuidado de la embarazada(12) y, en todo caso, se conoce la asociación directa entre los niveles de riesgo y sus efectos ${ }^{(8,12)}$.

Si bien, los trabajos de Frey et al. ${ }^{(13)}$ y de Bidstrup et al. ${ }^{(14)}$, no encontraron evidencia entre la exposición a peligros químicos y la duración del embarazo y tampoco con la hipertensión de la trabajadora embarazada(15), sí que existió en relación al bajo peso del neonato ${ }^{(16-18)}$. En este sentido, las trabajadoras sugirieron evitar los factores estresantes y la necesidad de mejorar la información/asesoramiento durante el embarazo(19) Y, apreciaron los consejos recibidos. De hecho, la utilización de algún tipo de material de protección personal evitó la exposición a las sustancias químicas y a las radiaciones(20,21). Jahnke et al. ${ }^{(22)}$, señalaron la importancia de la existencia de políticas de empresa relacionadas con la maternidad. Pero, el estudio de Herontin et al. ${ }^{(23)}$, concluyó que se realizaron escasas acciones preventivas y existió poca relación entre las empleadas y los servicios de salud.

En consecuencia y por lo anteriormente descrito, el objetivo de la presente revisión fue conocer y analizar los efectos derivados de la exposición laboral en las mujeres trabajadoras embarazadas expuestas a sustancias peligrosas. 


\section{Métodos}

\section{Diseño}

Estudio descriptivo transversal y análisis crítico de los trabajos recuperados mediante revisión sistemática.

La estructura de la presente revisión siguió la guía de comprobación para revisiones sistemáticas Preferred Reporting Items for Systematic reviews and Meta-Analyses (PRISMA).

\section{Fuente de obtención de los datos}

Los datos se obtuvieron de la consulta directa y acceso, vía Internet, a las siguientes bases de datos bibliográficas del ámbito de las ciencias de la salud: MEDLINE (vía PubMed), Embase, Cochrane Library, Scopus, Web of Science, Literatura Latinoamericana y del Caribe en Ciencias de la Salud (LILACS) y Medicina en Español (MEDES).

\section{Unidad de análisis}

Se trabajó con los artículos publicados y recuperados desde las bases de datos bibliográficas indicadas.

\section{Tratamiento de la información}

Para definir los términos de la búsqueda se consultó el Thesaurus de los Descriptores en Ciencias de la salud (DeCS) desarrollado por el Centro Latinoamericano y del Caribe de Información en Ciencias Médicas (BIREME) y su equivalencia con el establecido por la U.S. National Library of Medicine, los Medical Subject Headings $(\mathrm{MeSH})$.

Del estudio del jerárquico de ambos Thesaurus y de sus fichas de indización se consideraron adecuadas las siguientes ecuaciones de búsqueda:

a. Población - Embarazo (Pregnancy): Estado durante el que los mamíferos hembras llevan a sus crías en desarrollo -embrión o feto- en el útero, antes de nacer, desde la fertilización hasta el nacimiento.

"Pregnancy"[Mesh] OR "Pregnancy"[Title/Abstract] OR "Pregnancies"[Title/Abstract] OR "Gestation*"[Title/Abstract] OR "Pregnancy Complication"[Mesh] OR "Pregnancy Complication*"[Title/Abstract] OR "Child Bearing"[Title/Abstract] OR "Childbearing"[Title/Abstract] OR "Gestation*"Title/Abstract] OR “Gravidity"[Title/Abstract]

b. Intervención - Sustancias Peligrosas (Hazardous Substances): Elementos, compuestos, mezclas o soluciones que se consideran gravemente perjudiciales para la salud humana y el medio ambiente. Se incluyen las sustancias que son tóxicas, corrosivas, inflamables o explosivas. 
"Hazardous Substances"[Mesh] OR "Hazardous Substance*"[Title/Abstract] OR "Hazardous Material*”[Title/Abstract] OR "Hazardous Chemical*"[Title/Abstract] OR "Environmental Toxic Substance*"[Title/Abstract] OR "Toxic Environmental Substance*"[Title/Abstract] OR "Biohazard*”[Title/Abstract] OR “Dangerous Good*"[Title/Abstract]

c. Resultado - Exposición Profesional (Occupational Exposure): Exposición a agentes químicos, físicos o biológicos potencialmente dañinos que ocurre como resultado de la ocupación.

"Occupational Exposure"[Mesh] OR "Occupational Exposure*"[Title/Abstract] OR "Work Exposure*"[Title/Abstract] OR "Labor Exposure*"[Title/Abstract] OR "Occupational Exposition*"[Title/Abstract] OR "Work Exposition*"[Title/Abstract] OR "Labor Exposition*”[Title/Abstract] OR "Job Exposure*"[Title/Abstract] OR “Job Exposition*"[Title/Abstract]

Las ecuaciones de búsqueda finales se desarrollaron para su empleo en la base de datos MEDLINE, vía PubMed, mediante la unión booleana de las 3 ecuaciones propuestas: Población AND Intervención AND Resultado, utilizando los filtros: humanos "Humans" y adultos "Adult: 19+ years".

Esta estrategia se adaptó, posteriormente, a las características de cada una del resto de bases de datos consultadas.

La búsqueda desde la primera fecha disponible en cada una de las bases seleccionadas hasta el 26 de enero de 2021 y se completó con el examen del listado bibliográfico de los artículos que fueron seleccionados.

\section{Selección final de los artículos}

Se escogieron, para la revisión y análisis crítico, los artículos que cumplan los siguientes criterios:

- Inclusión: adecuarse a los objetivos de la búsqueda, ser artículo original, estar publicados en revistas arbitradas por pares y redactados en inglés, español o portugués.

- Exclusión: aquellos artículos que no se pudieron encontrar el texto completo, no exista relación entre la intervención y el resultado a estudio (criterio de causalidad: exposición laboral derivada por una sustancia peligrosa) y los que incluyan población no adulta (menores de 18 años).

La selección de artículos pertinentes se realizó por los autores de la presente revisión. Para dar por válida la inclusión de los artículos, se estableció que la valoración de la concordancia entre las tres autoras (índice Kappa) debía ser superior a $0,60^{(24)}$. Siempre que se cumpliera esta condición, las posibles discordancias se solucionarían mediante consenso entre todos los autores de la revisión. 


\section{Corrección documental, nivel de evidencia y grado de recomenda- ción}

La adecuación de los artículos seleccionados, desde el punto de vista de su corrección estrucutral, se valoró utilizando como apoyo las pautas para informar los estudios observacionales STROBE (STrengthening the Reporting of OBservational studies in Epidemiology)(25), que contiene una lista de 22 puntos de control esenciales que deben describirse durante la publicación de estos documentos. Para cada artículo seleccionado, se asignó un punto por cada ítem presente (en caso de no ser aplicable, no puntuó). Cuando un ítem estuvo compuesto por varios apartados, estos se evaluaron de forma independiente, dándole el mismo valor a cada uno de ellos y posteriormente se realizó un promedio (siendo éste el resultado final de ese ítem), de tal forma que en ningún caso se superó la valoración total de un punto por ítem.

Para conocer el nivel de evidencia y su grado de recomendación se usaron las recomendaciones del Scottish Intercollegiate Guidelines Network Grading Review Group (SIGN)(26).

\section{Extracción de los datos}

El control de la corrección de los datos se realizará mediante dobles tablas que permitieron la detección de las desviaciones y su subsanación mediante nueva consulta de los originales.

La depuración de los registros duplicados (presentes en más de una base de datos) se ejecutó mediante el programa multiplataforma ZOTERO (gestor de referencias bibliográficas desarrollado por el Center for History and New Media de la Universidad George Mason).

Para determinar la actualidad de los estudios, se calculó el semiperiodo de Burton-Kebler (BK) y el índice de Price (IP).

Los artículos se agruparon según las variables a estudio, con el fin de sistematizar y facilitar la comprensión de los resultados, considerando los siguientes datos: primer autor, año de publicación, tipo de estudio, población estudiada, país y periodo del estudio, agente(s) de exposición, resultado principal motivada por el efecto de la exposición.

\section{Análisis de los datos}

Los datos relacionados con la recuperación de la información se presentaron mediante su frecuencia y porcentaje.

Para conocer el BK se calculó la mediana de la edad según rango temporal analizado y el IP mediante el porcentaje de artículos con edad inferior a los 5 años.

La medida de la concordancia para conocer la pertinencia de la selección de los artículos se realizó mediante el IK. Se consideró valida esta relación entre autores 
cuando su valor fuera superior al 60\% (fuerza de la concordancia buena o muy buena).

Las puntuaciones del cuestionario STROBE se analizaron mediante la mediana, su máximo y su mínimo. La evolución de este puntaje, en relación a los años de publicación, se obtuvo mediante el análisis de correlación de Pearson.

\section{Aspectos éticos}

Todos los datos fueron obtenidos de los artículos aceptados para la revisión. Por tanto y conforme con la Ley 14/2007, de investigación biomédica(27), no fue necesaria la aprobación del Comité de Ética al utilizar datos secundarios.

\section{Resultados y discusión}

Al aplicar los criterios de búsqueda se recuperaron un total de 366 artículos: 128 (34,97\%) en MEDLINE (vía PubMed), 69 (18,85\%) en Embase, 2 (0,55\%) en Cochrane Library, 93 (25,41\%) en Scopus, 71 (19,40\%) en Web of Science y 3 (0,82\%) en LILACS. En la base bibliográfica MEDES no se recuperó ningún documento. La consulta de los listados bibliográficos de los artículos seleccionados permitió seleccionar 54 estudios.

Tras depurar los 118 registros repetidos y aplicar los criterios de inclusión y exclusión (figura 1), fue posible seleccionar 35 documentos ${ }^{(28-62)}$ para su revisión y análisis crítico; ver tabla 1.

El acuerdo sobre la pertinencia de los estudios seleccionados entre los evaluadores, calculado mediante el índice Kappa, fue del 70,00\% ( $p=0,01)$.

Los artículos seleccionados presentaron una obsolescencia, según el Índice de Burton-Kebler igual a 22,00 años, con un Índice de Price del 0,00\%. El año con mayor número de trabajos publicados fueron en el 1999, del cual se seleccionaron 3 artículos para la revisión(43-45).

La obsolescencia de los artículos relacionados fue algo mayor de lo esperado en el ámbito de las ciencias de la salud ${ }^{(63,64)}$. Pero, similar a lo encontrado en una anterior revisión sistemática relacionada con la salud laboral(65). Esto se confirma por el hecho de que todos los estudios aceptados datan de una fecha de publicación anterior a los 5 años, lo cual pone de manifiesto la necesidad de su actualización. 


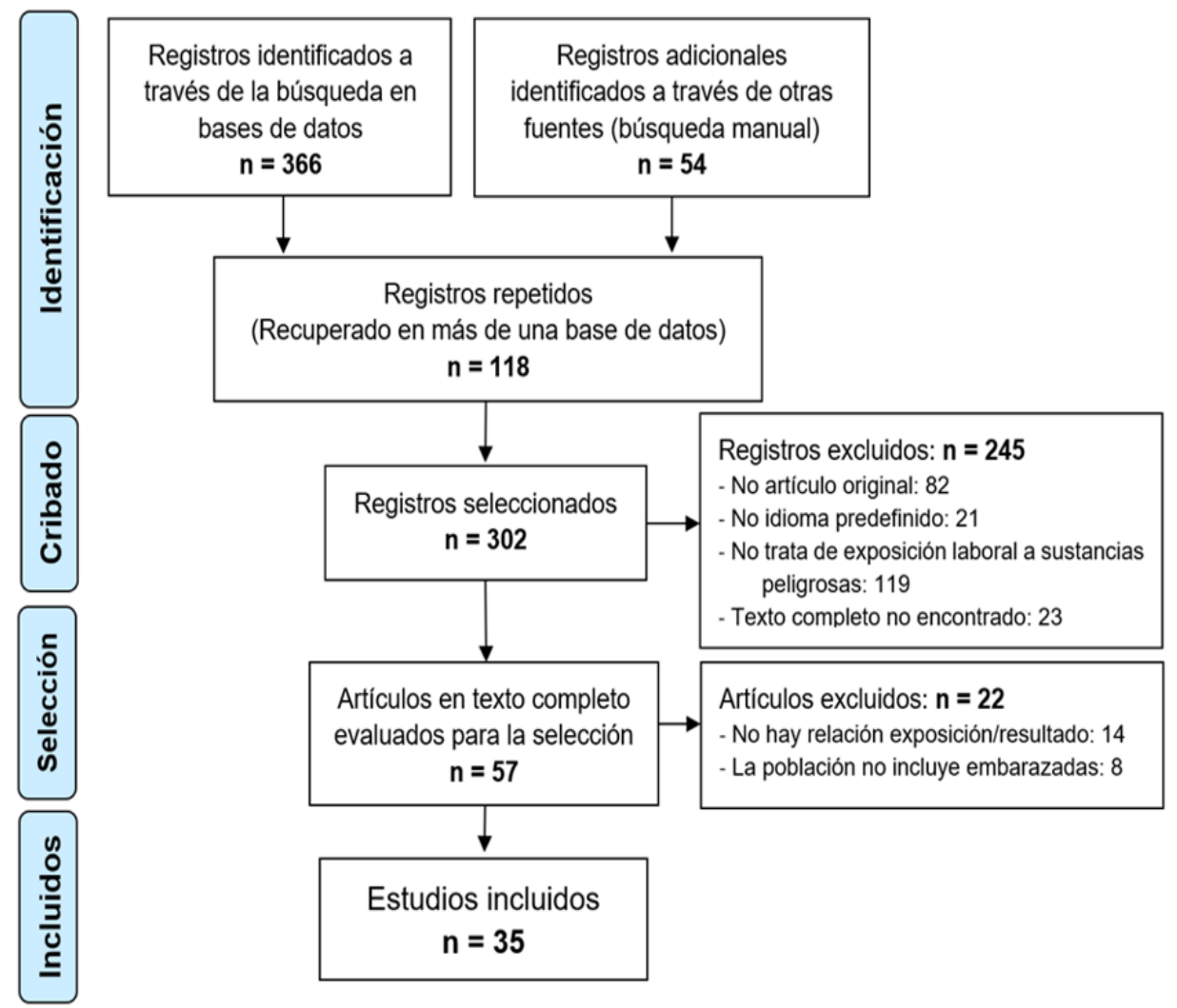

Figura 1: Diagrama de flujo de la identificación y selección de los artículos Al evaluar los estudios mediante el cuestionario STROBE, las puntuaciones oscilaron entre un mínimo de 7,50 y un máximo de 19,66 (sobre 21 ítems), con mediana igual a 15,32 (tabla 2), observando, a lo largo del tiempo, una moderada tendencia lineal creciente $\left(R^{2}=0,35 ; p<0,001\right)$.

La evaluación de la corrección documental de los estudios incluidos en este trabajo mediante STROBE resultó similar a la de otros artículos de revisión ${ }^{(63,65)}$. El estudio de la progresión en la adecuación documental que se observó en los artículos más actuales se debe fundamentalmente a la implantación de estos cuestionarios. De hecho, los trabajos más antiguos no solían seguir estas guías de calidad, por ejemplo, los primeros documentos sobre STROBE datan del año 2004 y su utilización fue de forma progresiva ${ }^{(66)}$. Habría que dejar constancia que en la gran mayoría de los estudios seleccionados no se especificaron todas las medidas adoptadas para afrontar fuentes potenciales de sesgo tampoco se describieron las razones de la pérdida de participantes en cada fase de la investigación o se realizaron análisis adicionales de interacciones o sensibilidad. Todo ello, es la consecuencia de no haber obtenido mayores puntajes.

Según los criterios SIGN esta revisión presentó evidencia 2+ (estudios de cohortes o de casos y controles bien realizados, con bajo riesgo de confusión, sesgos o azar y una moderada probabilidad de que la relación sea causal) con grado de 
recomendación C (un cuerpo de evidencia que incluya estudios directamente aplicables a la población objeto y que demuestren globalmente consistencia de los resultados).

Este resultado viene refrendado por el nivel de evidencia y grado de recomendación que obtendría el presente trabajo según los criterios SIGN que son acordes a las revisiones que se fundamentan en estudios observacionales. A pesar de buscar una consistente relación causa-efecto, ya que se buscaban trabajos de intervención, no hay que olvidar los diseños de los estudios de donde proviene la evidencia, asumiendo que algunos de ellos están sujetos a más sesgos que otros y por ende, justifican más débilmente las decisiones a tomar ${ }^{(67)}$. Además, estos criterios son los esperados para los diseños de los trabajos revisados.

Como bien denunciaron Correa et al.(68), la constante alusión a deficiencias metodológicas y conceptuales de los estudios observacionales y la falta de esfuerzos, por parte de los investigadores, para evaluar el impacto de sus conclusiones solo pueden crear un clima de confusión y escepticismo. En este mismo sentido, Vandenbroucke et al. ${ }^{(69)}$, diez años más tarde, señalaron que a menudo los informes de los estudios observacionales poseían una calidad insuficiente, por lo que el cumplimiento de las listas de recomendación que ayudan a mejorar la calidad de las publicaciones de tipo observacional, como STROBE, se hace cada vez más necesario.

Los trabajos revisados, fueron 13 estudios de cohortes $(30,31,35,37,38,44,45,47,48,51,52,58,62), 20$ estudios de casos y controles $(28,29,32,33,36,39-43,46,49,50,53,55-57,59,61)$ y 2 descriptivos transversales ${ }^{(34,54)}$.

Los países que aportaron un mayor número de investigaciones fueron Estados Unidos, con 7 estudios $(28,39,40,47,51,52,54)$ y Francia con $5^{(30,31,36,50,56)}$. Si bien, es destacable que se revisaron trabajos de 13 países diferentes y también se recopiló un estudio donde participaron varios países ${ }^{(42)}$.

El predominio de la filiación estadounidense es un hecho conocido y recogido ampliamente en la literatura científica. A ello contribuye la potencia de sus universidades y la importante financiación pública y privada de sus instituciones y centros de investigación. Dato que se relaciona íntimamente con el idioma de publicación, donde es conocido el predominio del inglés. Resultados nada novedosos, pues la omnipresencia del inglés es de sobra conocida y una constante en las publicaciones de las ciencias de la salud(70).

La edad de la población incluida fue mayor de los 18 años salvo los trabajos de Vaktskjold et al.(29), donde se indicaba que se incluían mujeres menores de esa edad; el de Halliday-Bell et al. ${ }^{(32)}$, en el que se indicaba que la población presentaba una edad de menos de 19 años hasta los 40 y el de Vaktskjold et al. ${ }^{(37)}$, del año 2006, donde se indicaba que las mujeres presentaban edad entre los 13 y 46 años. Ahora bien, hay que indicar que en un buen número de estudios no figuraba la edad de la población.

El trabajo que contó con una mayor población fue el de Halliday-Bell et al. ${ }^{(32)}$, con $n=31746$ mujeres. Los periodos estudiados fueron, en todos los casos, amplios 
(como mínimo de 1 año), aunque en el estudio de McAbee et al.(54), no figuraba el espacio temporal en el que se desarrolló la investigación.

Es cierto que las edades de las mujeres incluidas en los diferentes estudios podría considerarse altas, pero se corresponde con lo observado en una investigación anterior sobre trabajadoras embarazadas ${ }^{(71)}$.

Algunos artículos recuperados estudiaban población trabajadora menor y aunque se han incluido en la revisión, al presentar un tamaño poblacional donde existían prioritariamente mujeres adultas, hay que tener presente que existe consenso en que los niños/as, desde el nacimiento hasta el final de la adolescencia, son más vulnerables a los contaminantes químicos que los adultos. Esta susceptibilidad durante la infancia, junto con la sensibilidad biológica como característica inherente al crecimiento, puede producir daños irreparables en el desarrollo de sistemas y órganos que pueden llevar a la discapacidad e incluso a la muerte en edades tempranas. De hecho, una cuestión todavía no resuelta es en qué medida los contaminantes químicos dispersos en el medio ambiente pueden estar contribuyendo al cambio de patrón en las enfermedades pediátricas ${ }^{(72)}$.

En cuanto al período de seguimiento resultó adecuado para valorar los resultados de la intervención (efectos de la exposición), requisito que cumplieron todos los estudios seleccionados. Se considera necesario un periodo de varias semanas, incluso meses, para poder valorar los resultados ${ }^{(64,65)}$.

\section{Principales exposiciones observadas}

A la hora de revisar los artículos se observó que existían estudios que, si bien presentaron una misma población y exposición, los resultados ofrecidos eran distintos por lo que se optó por incluirlos en la tabla resumen en aras de recoger la máxima información posible. Este fue el caso de los trabajos Vaktskjold et al., de los años 2006 y $2008^{(33,37)}$, y de los de Shaw et al., de los años 2001 y $2003^{(39,40)}$.

La exposición más frecuente que se constató fue a los disolventes orgánicos, en 25 de los 35 estudios revisados ${ }^{(29-31,35,36,38,41-51,53,55-60,62)}$, utilizados en una gran variedad de trabajos (construcción, serigrafía, laboratorio, peluquería, etc.). Los disolventes más observados fueron: benceno( $29,35,38,44,53)$, tolueno(29,35,44,53,60), xileno(29,35,44,53,60), acetona ${ }^{(29,35,53)}$ y alcoholes alifáticos y aromáticos ${ }^{(35,44,56,60)}$; ver tabla 1.

Como se ha visto en esta revisión los disolventes son uno de los productos químicos industriales de mayor uso, producidos y utilizados en grandes cantidades, bajo una gran variedad de denominaciones comerciales y químicas, en casi todas las industrias. La mayoría contienen compuestos orgánicos volátiles que se evaporan fácilmente, son inflamables y pueden disolverse en grasas, por lo que el riesgo de exposición laboral y medioambiental durante su utilización es muy elevado (73). Por su volatilidad pueden ser inhalados, siendo la inhalación la principal vía de exposición laboral. 
Al mismo tiempo, otras sustancias como pesticidas, combustibles, barnices, tintes, detergentes, productos de peluquería, etc., también suelen contener en su formulación disolventes, lo que aumenta la probabilidad a esta exposición laboral.

Otras exposiciones que se comprobaron en esta revisión fueron a: gases anesté$\operatorname{sicos}^{(34,58,61)}$, pesticidas o herbicidas ${ }^{(28,34,57)}$, níquel soluble en agua ${ }^{(33,37)}$, plomo, mercurio y otros metales ${ }^{(42,55,58,60)}$, combustibles ${ }^{(36,55)}$, pinturas y barnices ${ }^{(41,50,55)}$, tintes y pigmentos ${ }^{(39,55,56)}$, propelentes ${ }^{(39)}$, detergentes ${ }^{(5,5,57,60)}$, productos de peluquería y estética $^{(32,42)}$, medicamentos antineoplásicos y $\operatorname{citostáticos}^{(42,61)}$ y radiaciones ${ }^{(34,54)}$; ver tabla 1.

En 3 estudios se identificó la exposición a una gran cantidad de compuestos químicos, especificándose, tan solo, que eran 74 o más ${ }^{(39,40,42)}$. En el trabajo de Irwin et al.(52), no se especificaba las sustancias peligrosas a las que estaban expuestas las trabajadoras.

Como se ha comprobado, una mayoría de los artículos revisados estudiaron diferentes sustancias de exposición, sin explicitar claramente los posibles factores de confusión que podrían haberse producido. Tampoco quedó muy claro las diferentes interacciones que existieron entre las diferentes sustancias químicas. En los estudios sobre exposición laboral se debe realizar un esfuerzo por medir adecuadamente el efecto de la confusión y la interacción de factores personales y laborales, como pueden ser el sexo, el trabajo doméstico, la antigüedad laboral, la carga de trabajo, los turnos y el tipo de contrato. Además, las investigaciones deben centrarse en esclarecer los mecanismos de relación entre los factores de exposición ${ }^{(74)}$.

La falta de información junto a la ausencia de un conocimiento preciso de las propiedades intrínsecas de cada agente químico y de la exposición derivada de un uso concreto dificultan en gran medida la prevención de los trabajadores expuestos a los riesgos generados por la presencia de estos productos en los puestos de trabajo(75). Por otro lado, no siempre fue evidente si las exposiciones fueron repetitivas o no.

La magnitud del problema y de la falta de control a la exposición a químicos en general ha llevado a que algunos investigadores piensen que el patrón epidemiológico de las enfermedades pueda estar cambiando en las sociedades desarrolladas si se observan las principales causas de mortalidad y morbilidad. Los contaminantes parecen estar reemplazando a los microbios y las enfermedades degenerativas a las infecciones. Incluso esta exposición puede estar influyendo en la aparición y distribución de enfermedades nuevas o desconocidas hasta hace poco tiempo ${ }^{(76)}$.

Las medidas necesarias para mejorar la prevención serían, en primer lugar, la elaboración de criterios de valoración de riesgos laborales durante el embarazo homogéneos según la exposición a los riesgos específicos y las semanas de gestación y, en segundo lugar, mejorar la información desde los servicios de prevención a las trabajadoras embarazadas y a las mujeres en edad fértil, tras la evaluación de los posibles peligros de cada puesto de trabajo(2,7). 


\section{Efectos derivados de las exposiciones}

El efecto más observado, derivado de la exposición de la trabajadora embarazada, fue la presencia de anomalías congénitas, notificado en 22 artícu$\operatorname{los}^{(28-31,36-40,42-44,46,47,50,53,56,58-62)}$. Las malformaciones congénitas más reportadas fueron: las hendiduras oro-faciales (labio leporino y paladar hendido), presentes en 7 estudios $(31,36,39,42,46,49,56)$. Otras anomalías fueron: los defectos del tubo neural(28,38,40), las malformaciones del tracto urinario( ${ }^{(31,60)}$, de los genitales masculinos ${ }^{(31,37)}$, del tubo digestivo(56) y anomalías conotruncales ${ }^{(39)}$. También se detectó, en la revisión de los trabajos aceptados, que 10 artículos reportaron la presencia de anomalías congénitas en general, sin centrarse en grupos concretos ${ }^{(29,30,43,44,47,53,58,59,61,62)}$. En 13 de los artículos se encontraron una asociación significativa entre las exposiciones y malformaciones congénitas ${ }^{(31,36,38,39,42-44,46,50,56,59-61)}$ y 9 de los artículos no mostraron un incremento significativo del riesgo de malformaciones ${ }^{(28-30,37,40,47,53,58,62)}$. En la tabla 3 se muestra la asociación de cada estudio con el químico específico.

El segundo efecto más observado fue el aborto espontáneo, reportado por 14 artí$\operatorname{culos}^{(30,33-35,48,49,51,53,54,57-59,61,62)}$. De este grupo, 8 artículos encontraron un incremento significativo del riesgo derivado de la exposición $\mathrm{n}^{(34,48,49,51,53,54,5,59)}$, mientras que 6 no encontraron una asociación significativa ${ }^{(30,33,35,58,61,62)}$; ver tabla 3.

Por último, se observó otros efectos señalados en algunos artículos, como: El parto pretérmino(32,35,47). Halliday-Bell et al.(32), encontraron un incremento significativo del riesgo (OR $=1,21$; IC95\% 1,07-1,38), mientras que Frey et al. ${ }^{(35)}$ y Hewitt et al. ${ }^{(47)}$, no encontraron asociación.

Otros 3 artículos observaron bajo peso al nacer(32,47,58). Halliday-Bell et al.(32), indicaron un incremento significativo del riesgo ( $O R=1,44$; IC95\% 1,23-1,69); por el contrario, Hewitt et al. ${ }^{(47)}$ y Ahlborg et al. ${ }^{(58)}$, no encontraron asociación.

En 4 estudios se constató muerte perinatal(32,35,58,62): Halliday-Bell et al. ${ }^{(32)}$ encontraron riesgo incrementado $(\mathrm{OR}=1,62$; IC95\% 1,01-1,60); por el contrario, Frey et al. ${ }^{(35)}$, Ahlborg et al. ${ }^{(58)}$ y Axelsson et al. ${ }^{(62)}$ no encontraron asociación.

También, en 3 trabajos, se observó hipertensión inducida por el embarazo(35,47,52): Hewitt et al. ${ }^{(47)}$ encontraron un incremento significativo (RR = 3,5; IC95\% 1,0-12,2). Pero, ninguna asociación fue encontrada por Frey et al. ${ }^{(35)}$ o Irwin et al. ${ }^{(52)}$.

Otros resultados obtenidos de la exposición que solo fueron indicados en un solo estudio pueden consultarse en la tabla 1.

El análisis de la evidencia encontrada en esta revisión mostró la asociación entre la exposición a las sustancias peligrosas, en diferentes situaciones de trabajo, con la aparición de daños para la salud de la trabajadora embarazada o su descendencia, situación que confirman los resultados de un anterior trabajo(75). Asimismo, Saavedra-Ontiveros et al.(77), relacionaron la contaminación industrial con solventes orgánicos como causa de teratogénesis. Y, como señalaron Cordier et al. ${ }^{(78)}$, aunque el nivel de evidencia de estos resultados y la no asociación observada, en algunas investigaciones, pudieran generar incertidumbre es patente que cada vez 
hay mayor certeza de que la exposición a determinados solventes sugiere relación causal entre la exposición y la aparición de alteraciones congénitas.

Por otro lado, el resultado de aborto espontáneo por exposición a sustancias peligrosas ya fue referido por el National Institute for Occupational Safety and Health $(\mathrm{NIOSH})^{(79)}$, que señalaba que a pesar de existir aun desconocimiento sobre las causas de la mayoría de los problemas de salud reproductiva si se puede asegurar que algunos peligros, en el lugar de trabajo, pueden afectar la salud reproductiva de la mujer, su capacidad de quedar embarazada, o la salud de los bebés en el vientre de la madre.

Pak et al. ${ }^{\left({ }^{80}\right)}$, reconociendo la necesidad de más investigaciones para comprender los riesgos reproductivos laborales, señalaban la necesidad de discutir las intervenciones para reducir los riesgos de trastornos reproductivos entre las poblaciones de trabajadoras susceptibles.

Igualmente, Delvigne et al.(81), en un reciente estudio, concluyeron que la exposición a contaminantes debía tenerse en cuenta en la asistencia sanitaria preventiva en el campo de la reproducción de acuerdo con la creciente cantidad de datos que se publican y corroboran las observaciones que subrayan la importancia de reforzar los conceptos de salud ambiental y laboral en la formación inicial y continua de los profesionales de la salud.

Ahora bien, no hay que olvidar lo manifestado por Kumar et al. (82), que teniendo en cuenta que si bien muchas personas están expuestas a factores químicos, físicos, biológicos, ambientales y ocupacionales, los datos relacionados con el papel de estos factores en la reproducción femenina son escasos. Pero, sugieren efectos adversos, de ciertos tóxicos entre ellos los disolventes orgánicos, en el sistema reproductor femenino con afectación en el embarazo. Por lo tanto, existe la necesidad de un programa de concientización y prevención sobre los efectos adversos de estos factores y el deterioro de la salud reproductiva femenina, el resultado del embarazo y el desarrollo de la descendencia, ya que algunas de estas sustancias químicas podrían afectar al feto en desarrollo en dosis muy bajas mediante un mecanismo disruptivo endocrino.

En este mismo sentido, Snijder et al.(83), concluían que a pesar de algunas incertidumbres en la base de la evidencia, aún puede ser prudente desaconsejar la exposición a determinadas sustancias en el lugar de trabajo para las parejas que intentan concebir.

\section{Limitaciones del estudio}

Los resultados de esta revisión están limitados por las carencias de cada trabajo incluido en la misma. Los diseños epidemiológicos de los estudios seleccionados en esta revisión (cohortes y casos y controles), aportan un nivel de evidencia y grado de recomendación que no permiten asegurar por completo la validez y fiabilidad de las observaciones realizadas ${ }^{(65,72)}$. Además, la mayoría de los estudios 
no especificaron si realizaron control de los factores de confusión que pudieran afectar a los resultados.

Otra importante limitación de la presente revisión fue la exclusión de 23 artículos por no haber podido recuperar el texto completo, a pesar de contar con la ayuda de la Biblioteca Nacional de Ciencias de la Salud (BNCS-ISCIII) y la Red de Bibliotecas Universitarias Españolas (REBIUN). Obviamente, hubiera sido interesante poder contar con los resultados de estos trabajos.

Por otro lado, de las bases de datos de Scopus y Web of Science se recuperaron muchos trabajos que finalmente fueron irrelevantes, lo que podría deberse a la falta de indexación (la búsqueda se realizó en formato de texto consultando el título, el resumen y las palabras clave) y la imposibilidad de limitar la búsqueda por tipo de artículo. Este alto "ruido" documental ya había sido enunciado en anteriores revisiones sistemáticas ${ }^{(64,84)}$.

\section{Conclusiones y perspectivas}

De acuerdo con las recomendaciones sobre los objetivos de una revisión sistemática ${ }^{(85)}$, la revisión actual sintetizó la información relevante relacionada con las exposiciones laborales de las trabajadoras embarazadas con la finalidad de poner a disposición de la comunidad científica información importante que pueda ayudar a promover intervenciones adecuadas para la protección de la maternidad.

Los trabajos revisados presentaron alta obsolescencia y un grado evidencia y recomendación que no permitió asegurar por completo la validez y fiabilidad de las observaciones realizadas.

El análisis de los resultados mostró la asociación entre la exposición a sustancias peligrosas con la aparición de diferentes daños para la salud de la trabajadora embarazada y su descendencia. La mayor exposición fue a los disolventes orgánicos y el efecto más observado las alteraciones congénitas.

Quizá una adecuada utilización de la licencia preventiva, generalmente infrautilizada, junto con el estudio de la idoneidad del puesto de trabajo y si fuera necesario la adaptación o el cambio, serían las medidas preventivas a considerar en la protección de la salud de la mujer embarazada.

En todo caso, incidir en la importancia de las políticas de empresa, relacionadas con la maternidad, en la prevención de los factores de exposición. 
Tabla 1: Resumen de los artículos aceptados para la revisión sobre los efectos derivados de la exposición laboral en las mujeres trabajadoras embarazadas expuestas a sustancias peligrosas

\begin{tabular}{|c|c|c|c|c|c|c|}
\hline Primer autor y año & Diseño & Población & País & Periodo & Exposición & Resultado principal \\
\hline $\begin{array}{l}\text { Makelarski et al. } \\
2014^{(28)}\end{array}$ & Casos y controles & $\begin{array}{l}\text { Tipo trabajo: no consta. } \\
N=3452 \\
\text { Edad }=<21 \text { a }>35 \\
\text { Gintervención } \\
n=502 \\
\text { Edad }=<21 \text { a }>35 \text { años } \\
\text { Gcontroles } \\
n=2950 \\
\text { Edad }=<21 \text { a }>35 \text { años }\end{array}$ & EE.UU. & 1997 a 2002 & Pesticidas & $\begin{array}{l}\text { Niños con defectos de tubo neural OR =0,9 (IC 95\% 0,7- } \\
\text { 1,1). }\end{array}$ \\
\hline $\begin{array}{l}\text { Vaktskjold et al. } \\
2017^{(29)}\end{array}$ & Casos y controles & $\begin{array}{l}\text { Tipo trabajo: albañiles (pintoras, } \\
\text { escayolistas, empapeladoras). } \\
N=\text { no consta } \\
\text { Edad = no consta (incluye < } 18 \text { años) } \\
\text { Gintervención } \\
n=597 \text { madres } \\
\text { Edad = no consta (incluye < } 18 \text { años) } \\
\text { Gcontrol } \\
n=\text { las madres de } 14951 \text { neonatos } \\
\text { (más de un nacimiento por madre) } \\
\text { Edad = no consta (incluye }<18 \text { años) }\end{array}$ & Rusia & 1973 a 2005 & $\begin{array}{l}\text { Disolventes orgánicos: acetona, } \\
\text { benceno, tolueno, xileno, disolvente } \\
\text { de Stoddard (10-20\% hidrocarburos } \\
\text { aromáticos) }\end{array}$ & 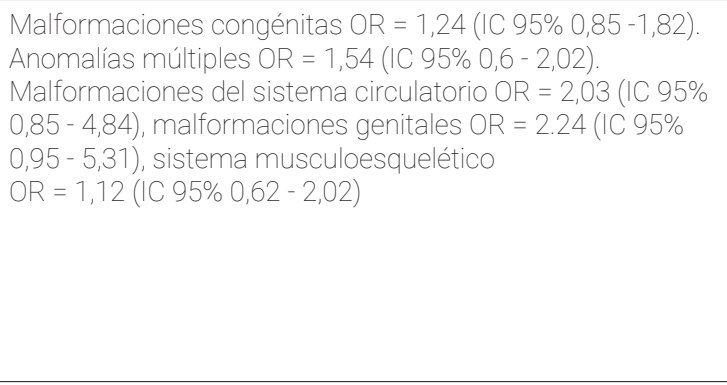 \\
\hline $\begin{array}{l}\text { Testud et al. } \\
\text { 2010(30) }\end{array}$ & $\begin{array}{l}\text { Cohortes } \\
\text { prospectivo }\end{array}$ & $\begin{array}{l}\text { Tipo trabajo: serigrafía, } \\
\text { microelectrónica, desengrasado de } \\
\text { metales, relojeríi industrial, laboratorios } \\
\text { biomédicos y de investigación, } \\
\text { tintorerías, pintura, talleres de muebles, } \\
\text { restauración de arte. } \\
N=412 \\
\text { Edad }=28,33 \pm 4,36 \\
\text { Gintervención } \\
n=206 \\
\text { Edad: } 28,33 \text { años } \\
\text { Gcontrol } \\
n=206 \\
\text { Edad }=28,22 \text { años }\end{array}$ & Francia & 1996 a 2008 & Disolventes orgánicos & $\begin{array}{l}\text { No se encontró asociación significativa entre la exposición } \\
\text { y riesgo de abortos espontáneos y malformaciones } \\
\text { congénitas ( } p>0,05 \text { ). } \\
\text { En el } 22,3 \% \text { de los casos se consideró exposición laboral } \\
\text { peligrosa para embarazo con recomendación de retirada de } \\
\text { su puesto de trabajo. } \\
\text { En el } 51,5 \% \text { de los casos la exposición no se consideró } \\
\text { peligrosa para el embarazo y no hubo cambios en } \\
\text { actividades profesionales. } \\
\text { En resto de los casos recomendaron cambios del puesto de } \\
\text { trabajo o medidas de protección. }\end{array}$ \\
\hline $\begin{array}{l}\text { Garlantézec et al. } \\
\text { 2009(31) }\end{array}$ & Cohortes & $\begin{array}{l}\text { Tipo trabajo: ocupaciones relacionadas } \\
\text { con ciencia oficina y servicios. } \\
N=3005 \\
\text { Edad = } 30 \text { años }\end{array}$ & Francia & 2002 a 2005 & Disolventes orgánicos & $\begin{array}{l}\text { Malformaciones congénitas (hendiduras orales, } \\
\text { malformaciones urinarias y malformaciones genitales } \\
\text { masculinas) OR =2,48 (IC } 95 \% 1,4-4,4 \text { ) para exposición } \\
\text { regular versus sin exposición; OR }=3,48 \text { (IC } 95 \% \text { 1,4-8,4 } \\
\text { para el nivel más alto de exposición frente a ninguna } \\
\text { exposición). }\end{array}$ \\
\hline $\begin{array}{l}\text { Halliday-Bell et al. } \\
2009^{(32)}\end{array}$ & Casos y controles & $\begin{array}{l}\text { Tipo trabajo: peluqueras, esteticistas. } \\
N=31746 \\
\text { Edad }=<19 \text { a }>40 \text { años } \\
\text { Gintervención } \\
n=13112 \\
\text { Edad }=<19 \text { a }>0 \text { años } \\
\text { Gcontroles } \\
n=18594 \\
\text { Edad }=<19 \text { a }>40 \text { años }\end{array}$ & Finlandia & 1990 a 2004 & Productos de peluquería y estética & 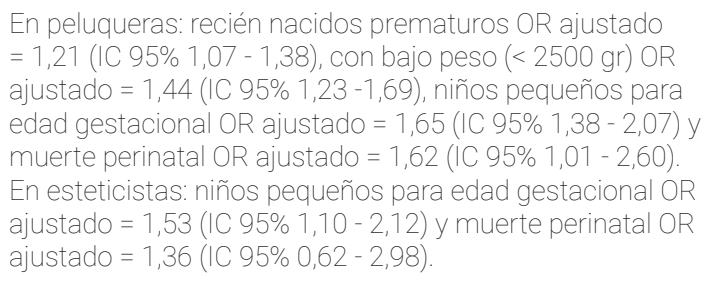 \\
\hline
\end{tabular}




\begin{tabular}{|c|c|c|c|c|c|c|}
\hline Primer autor y año & Diseño & Población & País & Periodo & Exposición & Resultado principal \\
\hline $\begin{array}{l}\text { Vaktskjold et al. } \\
2008^{(33)}\end{array}$ & Casos y controles & $\begin{array}{l}\text { Tipo trabajo: trabajadoras de refinería } \\
\text { de níquel. } \\
N=5045 \\
\text { Edad }=\text { no consta } \\
\text { Gintervención } \\
n=474 \\
\text { Edad }=25,3 \text { años } \\
\text { Gcontrol } \\
n=4571 \\
\text { Edad }=24,3 \text { años }\end{array}$ & Rusia & 1996 a 2002 & Níquel fácilmente soluble en agua & $\begin{array}{l}\text { Abortos espontáneos OR ajustado = 1,14 (IC 95\% 0,95 - } \\
\text { 1,37). }\end{array}$ \\
\hline $\begin{array}{l}\text { Shirangi et al. } \\
2008^{(34)}\end{array}$ & $\begin{array}{l}\text { Descriptivo } \\
\text { transversal }\end{array}$ & $\begin{array}{l}\text { Tipo trabajo: veterinarias. } \\
N=442 \\
\text { Edad media }=39,8 \text { años } \\
\text { Gintervención } \\
n=442 \\
\text { Edad media = } 39,8 \text { años } \\
\text { Gcontrol } \\
n=\text { no consta } \\
\text { Edad = no consta }\end{array}$ & Australia & 1960 a 2000 & $\begin{array}{l}\text { Gases anestésicos durante cirugías, } \\
\text { exposición a pesticidas, exposición } \\
\text { a rayos } X\end{array}$ & $\begin{array}{l}\text { Aborto espontáneo en las expuestas a gases anestésicos } \\
\text { sin extractor de gases OR }=2,49(\mathrm{IC} 95 \% 1,02-6,04) \text {, en } \\
\text { las expuestas a pesticidas OR=1,88 (IC } 95 \% 1,18-3,00) \text {, } \\
\text { radiaciones ionizantes } O R=1,82(\text { IC } 95 \% 1,17-2,82)\end{array}$ \\
\hline $\begin{array}{l}\text { Frey et al. } \\
2007^{(35)}\end{array}$ & $\begin{array}{l}\text { Cohortes } \\
\text { prospectivo }\end{array}$ & $\begin{array}{l}\text { Tipo trabajo: laboratorio o servicio } \\
\text { ténico. } \\
N=1047 \\
\text { Edad media }=30,6 \text { años } \\
N=1047 \text { madres } \\
\text { Edad media }=39,8 \text { años } \\
\text { Gintervención } \\
n=444 \\
\text { Edad }=\text { no consta } \\
\text { Gcontrol (trabajadoras de oficina) } \\
n=603 \\
\text { Edad }=\text { no consta }\end{array}$ & Alemania & 1997 a 2002 & $\begin{array}{l}\text { Disolventes orgánicos, sustancias } \\
\text { químicas para la investigación y } \\
\text { cancerígenos: acetona, metanol, } \\
\text { etanol, tolueno, dimetil formamida, } \\
\text { acetonitrilo, tetrahidrofurano, } \\
\text { diclorometano, xileno, ciclohexanona, } \\
\text { n-hexano, etil acetato, formaldehído, } \\
\text { alcohol isopropilico, n-propanol, ácido } \\
\text { sulfúrico, isobutanol }\end{array}$ & $\begin{array}{l}\text { Pérdida fetal: No se encontró asociación significativa con la } \\
\text { exposición a las sustancias a estudio. } \\
\text { Complicaciones del embarazo: } \\
\text { la exposición a tolueno y sangrado vaginal RR = 2,0 (IC95\% } \\
=1,0-4,1) \text {. } \\
\text { No asociación significativa entre exposición y nauseas o } \\
\text { HTA asociada al embarazo. } \\
\text { Duración del embarazo: no asociación significativa entre } \\
\text { exposición y nacimiento pretérmino. }\end{array}$ \\
\hline $\begin{array}{l}\text { Chevrier et al. } \\
2006^{(36)}\end{array}$ & Casos y controles & $\begin{array}{l}\text { Tipo trabajo: peluqueras-esteticistas, } \\
\text { trabajadoras industriales, } \\
\text { empaquetadores, ingenieras de } \\
\text { laboratorios, trabajadores de oficina- } \\
\text { secretarias, cajeras, dependientas. } \\
N=476 \\
\text { Edad media = } 29,6 \text { años } \\
\text { Gintervención } \\
n=240 \\
\text { Edad media }=29,5 \\
\text { Gcontrol } \\
n=236 \\
\text { Edad media }=29,7 \text { años }\end{array}$ & Francia & 1998 a 2001 & $\begin{array}{l}\text { Disolventes orgánicos: disolventes } \\
\text { oxigenados (alcoholes alifáticos, } \\
\text { aldehídos alifáticos, ésteres alifáticos, } \\
\text { cetonas alifáticas y éteres de glicol); } \\
\text { disolventes clorados (alcanos y } \\
\text { alquenos); y productos petrolfferos } \\
\text { (hidrocarburos aromáticos, mineral } \\
\text { spirits y combustible) }\end{array}$ & 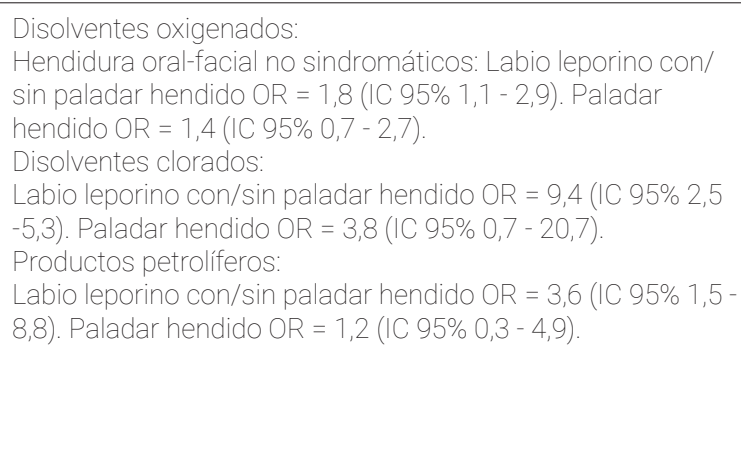 \\
\hline $\begin{array}{l}\text { Vaktskjold et al. } \\
2006^{(37)}\end{array}$ & Cohortes & $\begin{array}{l}\text { Tipo trabajo: trabajadoras de refinería } \\
\text { de níquel. } \\
N=23141 \\
\text { Edad media }=25 \text { años } \\
\text { Gintervención } \\
n=2942 \\
\text { Edad }=\text { no consta } \\
\text { Gcontrol } \\
n=20199 \\
\text { Edad }=\text { no consta }\end{array}$ & Rusia & 1998 a 2001 & Níquel fácilmente soluble en agua. & $\begin{array}{l}\text { Recién nacidos con malformaciones genitales } \mathrm{OR}=0,81 \text { (IC } \\
95 \% 0,52-1,26) \text {; con testículos no descendidos } \mathrm{OR}=0,76 \\
(\mathrm{IC} 95 \% \text { 0,40-1,47). }\end{array}$ \\
\hline
\end{tabular}




\begin{tabular}{|c|c|c|c|c|c|c|}
\hline Primer autor y año & Diseño & Población & País & Periodo & Exposición & Resultado principal \\
\hline $\begin{array}{l}\text { Wennborg et al. } \\
\text { 2005(38) }\end{array}$ & Cohortes & $\begin{array}{l}\text { Tipo trabajo: trabajadoras de } \\
\text { laboratorios. } \\
N=1629 \\
\text { Ginterveción (expuestos): } n=959 \\
\text { Edad = no consta } \\
\text { G control (no expuestos): } n=670 \\
\text { Edad no consta }\end{array}$ & Suecia & 1970 a 1989 & $\begin{array}{l}\text { Exposición a benceno y otros } \\
\text { disolventes }\end{array}$ & $\begin{array}{l}\text { Malformaciones, por exposición a disolvente antes del tercer } \\
\text { trimestre: OR = 1,8 (IC } 95 \% 1,0 \text { - 2,9). } \\
\text { Para el trabajo de laboratorio en general: OR = 1,2 (IC 95\% } \\
0,7-2,0) \text {. } \\
\text { Exposición a benceno alrededor de la concepción/ } \\
\text { organogénesis y defectos del tubo neural OR = 5,3 (IC 95\% } \\
1,4-21,1) \text {. } \\
\text { Hubo una mayor proporción de defectos del tubo neural en } \\
\text { relación con los disolventes, especialmente el benceno. }\end{array}$ \\
\hline $\begin{array}{l}\text { Shaw et al. } \\
2003^{(39)}\end{array}$ & Casos y controles & $\begin{array}{l}\text { Tipo trabajo: no consta. } \\
N=1768 \\
\text { Edad = no consta } \\
\text { G. intervención } \\
\text { Madres niños con hendidura orofacial } \\
n=662 ; \\
\text { malformaciones conotruncales } \\
n=207 \text {; } \\
\text { malformaciones de extremidades } \\
n=165 \\
\text { Edad }=\text { no consta } \\
\text { Gcontrol } \\
n=734 \\
\text { Edad }=\text { no consta }\end{array}$ & EE.UU. & 1987 a 1988 & 74 grupos agentes químicos & 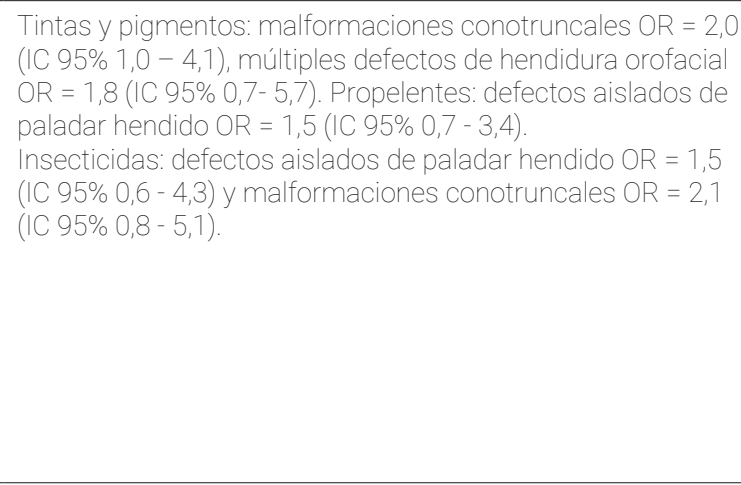 \\
\hline $\begin{array}{l}\text { Shaw et al. } \\
2007(40)\end{array}$ & Casos y controles & $\begin{array}{l}\text { Tipo trabajo: no consta. } \\
\text { Parte } 1 \\
N=1077 \\
\text { Edad = no consta } \\
\text { Gintervención } \\
n=538 \\
\text { Edad = no consta } \\
\text { Gcontrol } \\
n=539 \\
\text { Edad = no consta } \\
\text { Parte } 2 \\
N=746 \\
\text { Edad = no consta } \\
\text { Gintervención } \\
n=265 \\
\text { Edad = no consta } \\
\text { Gcontrol } \\
n=481 \\
\text { Edad = no consta }\end{array}$ & EE.UU. & $\begin{array}{l}\text { Parte 1: } \\
1989 \text { a } 1991 \\
\text { Parte 2: } \\
1987 \text { a } 1988\end{array}$ & $\begin{array}{l}74 \text { grupos de químicos } \\
\text { ocupacionales }\end{array}$ & $\begin{array}{l}\text { Parte 1: asociación significativa }(O R \geq 5) \text { entre defectos del } \\
\text { tubo neural en el recién nacido y exposición periconcepcional } \\
\text { ocupacional a } 12 \text { grupos de sustancias y sus combinaciones } \\
\text { (hidrocarburos aromáticos, sulfuros y disulfuros, orgánicos } \\
\text { halogenados, peróxidos, aminas aromáticas, hidrocarburos } \\
\text { alifáticos } C 1-4 \text {, hidrocarburos alifáticos C5-12, alcoholes } \\
\text { alifáticos, surfactantes, amoníaco e hidróxido de amonio, } \\
\text { aldehídos y dióxido de carbono) } \\
\text { Parte 2: no se encontró asociación significativa ( } O R<2,0) \\
\text { entre defectos del tubo neural y exposición a químicos de } \\
\text { estos } 12 \text { grupos, tanto aislados como en combinación. }\end{array}$ \\
\hline $\begin{array}{l}\text { Schüz et al. } \\
2000^{(41)}\end{array}$ & Casos y controles & $\begin{array}{l}\text { Tipo trabajo: no consta. } \\
N=4100 \\
\text { Edad }=\text { no consta } \\
\text { Gintervención } \\
n=1138 \\
\text { Edad }=\text { no consta } \\
\text { Gcontrol } \\
n=2962 \\
\text { Edad }=\text { no consta }\end{array}$ & Alemania & 1992 a 1997 & $\begin{array}{l}\text { Disolventes, pinturas y/o barnices, } \\
\text { derivados del petróleo, vapores } \\
\text { de plásticos y resinas, polvos } \\
\text { industriales, productos del fundido de } \\
\text { metales }\end{array}$ & $\begin{array}{l}\text { Leucemia linfocítica aguda en niños nacidos de } \\
\text { madres expuestas a pinturas y barnices en el periodo } \\
\text { preconcepcional } O R=1,6 \text { (IC } 95 \% \text { 1, - } 2,4) \text {, durante el } \\
\text { embarazo } O R=2,0(\text { (IC } 95 \% 1,2-2,3) \text { y en el periodo } \\
\text { postnatal } O R=1,0 \text { (IC } 95 \% \text { 0 } 0,6-1,8) \text {. No hubo asociación } \\
\text { significativa con el resto de químicos. }\end{array}$ \\
\hline
\end{tabular}




\begin{tabular}{|c|c|c|c|c|c|c|}
\hline Primer autor $y$ año & Diseño & Población & País & Periodo & Exposición & Resultado principal \\
\hline $\begin{array}{l}\text { Lorente et al. } \\
2000^{(42)}\end{array}$ & Casos y controles & $\begin{array}{l}\text { Tipo trabajo: trabajadoras de oficina, } \\
\text { servicio, agricultura y producción. } \\
N=851 \\
\text { Edad }=<24 \text { a }>35 \text { años } \\
\text { Gintervención } \\
n=100 \\
\text { Edad }=<24 \text { a }>35 \text { años } \\
\text { Gcontrol } \\
n=751 \\
\text { Edad }=<24 \text { a }>35 \text { años }\end{array}$ & $\begin{array}{l}\text { Francia, } \\
\text { Países Bajos, } \\
\text { Inglaterra, Italia }\end{array}$ & 1989 a 1992 & 314 agentes químicos & 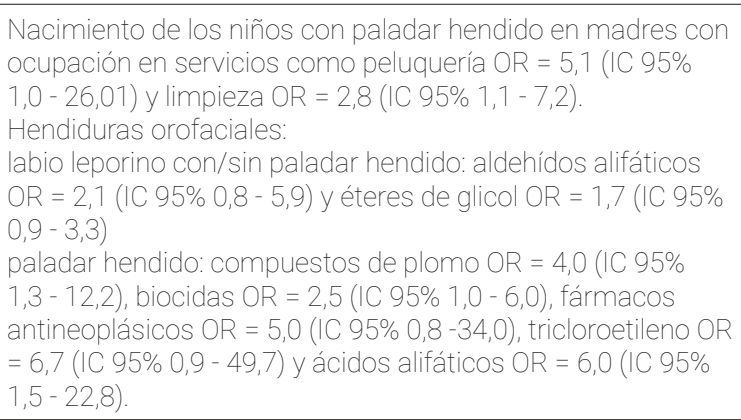 \\
\hline $\begin{array}{l}\text { Hrubá et al. } \\
\text { 1999(43) }\end{array}$ & Casos y controles & $\begin{array}{l}\text { Tipo trabajo: no consta. } \\
\text { Gintervención } \\
n=3897 \\
\text { Edad = No consta } \\
\text { Gcontrol } \\
\text { No constan datos }\end{array}$ & $\begin{array}{l}\text { República } \\
\text { Checa }\end{array}$ & 1990 a 1992 & Disolventes orgánicos. & $\begin{array}{l}\text { Malformaciones congénitas en niños mujeres expuestas a } \\
\text { disolventes orgánicos durante el } 2^{\circ} \text { trimestre de embarazo, } \\
\mathrm{OR}=2,37 \text { ( } p<0,05) \text {. }\end{array}$ \\
\hline $\begin{array}{l}\text { Khattak et al. } \\
1999(44)\end{array}$ & Cohortes & $\begin{array}{l}\text { Tipo trabajo: trabajadoras industriales, } \\
\text { ténicos de laboratorio, artistas, } \\
\text { industria de impresión, otros. } \\
N=250 \\
\text { Edad }=\text { no consta } \\
\text { Gintervención } \\
n=125 \\
\text { Edad media }=29,4 \text { años } \\
\text { Gcontrol } \\
n=125 \\
\text { Edad media }=29,5 \text { años }\end{array}$ & Canadá & 1987-1986 & $\begin{array}{l}\text { Disolventes orgánicos: hidrocarburos } \\
\text { alifáticos y aromáticos, fenol, } \\
\text { tricloroetileno, xileno, cloruro de vinilo, } \\
\text { acetona y otros }\end{array}$ & $\begin{array}{l}\text { Niños con malformaciones congénitas en mujeres } \\
\text { expuestas RR }=13,0 \text { (IC 95\% 1,8 - 99,5). }\end{array}$ \\
\hline $\begin{array}{l}\text { Seidler et al. } \\
\text { 1999(45) }\end{array}$ & $\begin{array}{l}\text { Cohortes } \\
\text { prospectivo }\end{array}$ & $\begin{array}{l}\text { Tipo trabajo: no consta. } \\
N=3946 \\
\text { Edad }=<19 \text { a }>35 \text { años }\end{array}$ & Alemania & 1987 a 1988 & $\begin{array}{l}\text { Disolventes orgánicos, cloruro de } \\
\text { carbono, herbicidas, clorofenol, } \\
\text { bifenilos policlorados, aminas } \\
\text { aromáticas, plomo, mercurio }\end{array}$ & $\begin{array}{l}\text { Niños pequeños para edad gestacional: exposición a } \\
\text { clorofenol OR = 7,0 (IC } 95 \% 1,2-43,0) \text {, aminas aromáticas } \\
\text { exposición alta OR = 7,1 (IC 95\% 1,2-43,4), mercurio OR = } \\
\text { 1,8 (IC } 95 \% 1,1-2,8) \text {. }\end{array}$ \\
\hline $\begin{array}{l}\text { García et al. } \\
\text { 1998(46) }\end{array}$ & Casos y controles & $\begin{array}{l}\text { Tipo trabajo: trabajadoras del cuero. } \\
N=532 \\
\text { Edad = no consta } \\
\text { Gintervención } \\
n=261 \\
\text { Edad = no consta } \\
\text { Gcontrol } \\
n=261 \\
\text { Edad = no consta }\end{array}$ & España & 1993 a 1994 & Disolventes orgánicos & $\begin{array}{l}\text { Hendeduras orales-faciales OR }=6,18 \text { (IC 95\% 1,48 - 25,69). } \\
\text { Malformaciones de sistema nervioso OR }=1,02 \text { (IC 95\% } \\
0,12-8,51) \text {, cardiopatías congénitas OR }=1,78(\text { IC } 95 \% 0,44 \\
-7,17) \text {, malformaciones genitales OR }=4,05(\text { IC } 95 \% 0,77 \text { - } \\
21,44) \text {, anomalías múltiples OR }=3,14 \text { (IC } 95 \% 0,82-12,00)\end{array}$ \\
\hline $\begin{array}{l}\text { Hewitt et al. } \\
\text { 1998(47) }\end{array}$ & Cohortes histórico & $\begin{array}{l}\text { Tipo trabajo: no consta. } \\
N=370 \\
\text { Edad = no consta } \\
\text { Gintervención } \\
n=118 \\
\text { Edad }=19 \text { a } 43 \text { años } \\
\text { Gcontrol } \\
n=226 \\
\text { Edad }=14 \text { a } 39 \text { años }\end{array}$ & EE.UU. & 1990 a 1993 & Disolventes & 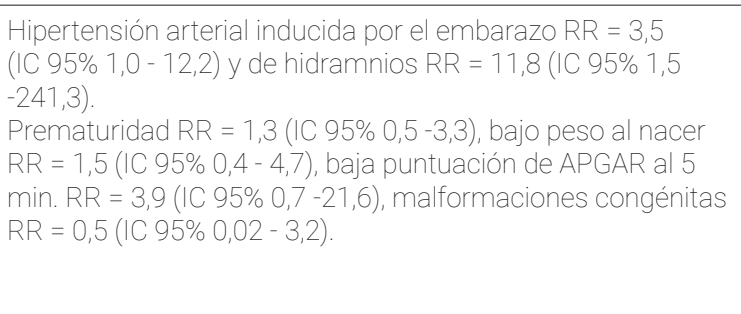 \\
\hline
\end{tabular}




\begin{tabular}{|c|c|c|c|c|c|c|}
\hline Primer autor y año & Diseño & Población & País & Periodo & Exposición & Resultado principal \\
\hline $\begin{array}{l}\text { Doyle et al. } \\
\text { 1997(48) }\end{array}$ & $\begin{array}{l}\text { Cohortes } \\
\text { retrospectivo }\end{array}$ & $\begin{array}{l}\text { Tipo trabajo: trabajadoras de lavandería } \\
\text { y de limpieza en seco. } \\
N=1748 \\
\text { Edad }=16 \text { a } 45 \text { años }\end{array}$ & Reino Unido & 1995-1996 & Percloroetileno & $\begin{array}{l}\text { Abortos espontáneos en las trabajadoras de limpieza en } \\
\text { seco expuestas OR = 1,67 (IC 95\% 1,17-2,36). }\end{array}$ \\
\hline $\begin{array}{l}\text { Agnesi et al. } \\
\text { 1997(49) }\end{array}$ & Casos y controles & $\begin{array}{l}\text { Tipo trabajo: trabajadoras de la } \\
\text { industria del zapato. } \\
N=216 \\
\text { Edad }=\text { no consta } \\
\text { Gintervención } \\
n=108 \\
\text { Edad }=\text { no consta } \\
\text { Gcontrol } \\
n=108 \\
\text { Edad }=\text { no consta }\end{array}$ & Italia & 1987 a 1988 & Disolventes orgánicos & $\begin{array}{l}\text { Abortos espontáneos en las trabajadoras expuestas a altas } \\
\text { concentraciones de disolventes orgánicos RR }=3,85 \text { (IC } \\
95 \% 1,24-11,9) \text {, exposición a bajas concentraciones de } \\
\text { disolventes orgánicos RR }=1,58 \text { (IC 95\% 0,62 -4,06). }\end{array}$ \\
\hline $\begin{array}{l}\text { Laumon et al. } \\
\text { 1996(50) }\end{array}$ & Casos y controles & $\begin{array}{l}\text { Tipo trabajo: no consta. } \\
N=600 \\
\text { Edad = no consta } \\
\text { Gintervención } \\
n=200 \\
\text { Edad = no consta } \\
\text { Gcontrol } \\
n=400 \\
\text { Edad }=\text { no consta }\end{array}$ & Francia & 1985 a 1989 & $\begin{array}{l}9 \text { subcategorías de disolventes } \\
\text { orgánicos, pinturas, pegamentos }\end{array}$ & $\begin{array}{l}\text { Hendiduras orales-faciales con exposición a disolventes } \\
\text { orgánicos en general OR }=1,62 \text { (IC } 95 \% 1,04-2,52) \text {, } \\
\text { alifáticos halogenados } O R=4,40 \text { (IC 95\% 1,41-16,15). }\end{array}$ \\
\hline $\begin{array}{l}\text { Swan et al. } \\
\text { 1995(51) }\end{array}$ & Cohortes & $\begin{array}{l}\text { Tipo trabajo: trabajadoras de fábrica. } \\
N=891 \\
\text { Edad }=18 \text { a } 44 \text { años }\end{array}$ & EE.UU. & 1986 a 1989 & $\begin{array}{l}\text { Disolventes fotorresistentes y } \\
\text { reveladores, compuestos de fluoruro, } \\
\text { disolventes de limpieza y dopantes }\end{array}$ & Abortos espontáneos RR = 3,21 (IC 95\% 1,29 - 5,96) \\
\hline $\begin{array}{l}\text { Irwin et al. } \\
\text { 1994(52) }\end{array}$ & Cohortes & $\begin{array}{l}\text { Tipo trabajo: militares embarazadas sin } \\
\text { hipertensión previa. } \\
N=6256 \\
\text { Edad }=>17 \text { años }\end{array}$ & EE.UU. & 1987 a 1989 & Agentes químicos no especificados & Hipertensión arterial inducida por el embarazo $(\mathrm{RR}=0,68)$ \\
\hline $\begin{array}{l}\text { Taskinen et al. } \\
\text { 1994(53) }\end{array}$ & $\begin{array}{l}\text { Casos-controles } \\
\text { retrospectivo }\end{array}$ & $\begin{array}{l}\text { Tipo trabajo: laboratorio. } \\
\text { Edad }=20-34 \\
N \text { total }=1176 \\
N \text { aborto }=535 \text { ( } 206 \text { casos, } 329 \\
\text { controles) } \\
N \text { malformaciones }=141 \text { ( } 36 \text { casos, } \\
105 \text { controles) } \\
N \text { controles por peso }=500\end{array}$ & Finlandia & 1970-1986 & $\begin{array}{l}\text { Acetona, acetonitrilo, benceno, } \\
\text { tetracloruro de carbono, cloroformo, } \\
\text { ciclohexano, etanol, éter, acetato de } \\
\text { etilo, formalina, heptano, isopropanol, } \\
\text { metanol, cloruro de metileno, } \\
\text { benceno de petróleo, tolueno, } \\
\text { tricloroetileno y xileno }\end{array}$ & 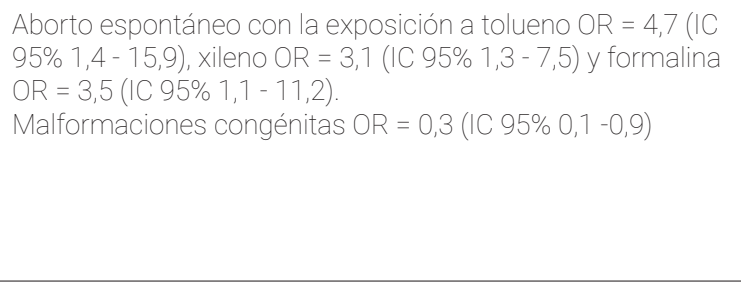 \\
\hline $\begin{array}{l}\text { McAbee et al. } \\
\text { 1993(54) }\end{array}$ & $\begin{array}{l}\text { Descriptivo } \\
\text { transversal }\end{array}$ & $\begin{array}{l}\text { Tipo trabajo: enfermeras de oncología. } \\
N=663 \\
\text { Edades medias: } \\
\text { 1er embarazo: } 24.7 \text { años } \\
2^{\circ} \text { embarazo: } 27,2 \text { años } \\
\text { 3er embarazo } 28,8 \text { años } \\
n=205 \text { enfermeras oncología } \\
n=226 \text { enfermeras no oncología } \\
n=232 \text { trabajadoras de la universidad }\end{array}$ & EE.UU. & No consta & $\begin{array}{l}\text { Control de la radiación y medir la } \\
\text { exposición crónica a la radiación a } \\
\text { dosis bajas }\end{array}$ & $\begin{array}{l}\text { Mortalidad neonatal y abortos espontáneos relacionados } \\
\text { con radiaciones ionizantes y agentes quimioterapéuticos OR } \\
=3,78(\text { IC } 95 \% 1,12-12,78) \text {. }\end{array}$ \\
\hline
\end{tabular}




\begin{tabular}{|c|c|c|c|c|c|c|}
\hline Primer autor y año & Diseño & Población & País & Periodo & Exposición & Resultado principal \\
\hline $\begin{array}{l}\text { Roeleveld et al. } \\
\text { 1993(55) }\end{array}$ & Casos y controles & $\begin{array}{l}\text { Tipo trabajo: trabajadoras en general. } \\
N=621 \\
\text { Edad = no consta } \\
\text { Gintervención } \\
n=306 \\
\text { Edad }=\text { no consta } \\
\text { Gcontrol } \\
n=315 \\
\text { Edad = no consta }\end{array}$ & Países Bajos & 1979-1987 & $\begin{array}{l}\text { Plomo, mercurio, arsénico, } \\
\text { disolventes orgánicos, combustible } \\
\text { diésel, hidrocarburos aromáticos } \\
\text { policíclicos, aminas aromáticas, } \\
\text { clorofenoles, bifenilos policlorados, } \\
\text { nitratos, formaldehído, óxido de } \\
\text { etileno, pinturas, tintes y pigmentos, } \\
\text { tintas, adhesivos, ceras y barnices, } \\
\text { herbicidas, detergentes, polvo } \\
\text { biológicamente activo, polvo de } \\
\text { madera/carbón/textil }\end{array}$ & $\begin{array}{l}\text { Retraso de desarrollo mental en los nacidos de trabajadoras } \\
\text { expuestas a pinturas, tintes y pigmentos } \mathrm{OR}=3,9(\mathrm{IC} 95 \% \\
1,0 \text { - 15,1) y a combustible diésel } \mathrm{OR}=2,0 \text { (IC } 95 \% \text { 1,0 - } 4,1) \text {. } \\
\text { No se encontró asociación significativa con el resto de los } \\
\text { químicos. }\end{array}$ \\
\hline $\begin{array}{l}\text { Cordier et al. } \\
\text { 1992 }\end{array}$ & Casos y controles & $\begin{array}{l}\text { Tipo trabajo: no consta. } \\
N=650 \\
\text { Edad }=\leq 35 \text { a }>35 \text { años } \\
\text { Gintervención } \\
n=325 \\
\text { Edad }=\leq 35>35 \text { años } \\
\text { Gcontrol } \\
n=325 \\
\text { Edad }=\leq 35 \text { a > } 35 \text { años }\end{array}$ & Francia & 1984 a 1987 & $\begin{array}{l}\text { Disolventes orgánicos "puros" ("White } \\
\text { spirit" tricloroetileno, alcoholes, } \\
\text { éteres, etc.) } \\
\text { y productos que normalmente } \\
\text { contienen disolventes (pinturas, } \\
\text { pegamentos, barnices, tintes, tintas y } \\
\text { similares). }\end{array}$ & $\begin{array}{l}\text { Hendiduras orales OR }=7,9 \text { (IC 95\% 1,8 - 44,9), } \\
\text { malformaciones del tubo digestivo OR }=11,9(\text { IC } 95 \% 2,0 \text { - } \\
\text { 149) y malformaciones múltiples OR }=4,5 \text { (IC 95\% 1,4-16,9). }\end{array}$ \\
\hline $\begin{array}{l}\text { Goulet et al } \\
1991^{(57)}\end{array}$ & Casos y controles & $\begin{array}{l}\text { Tipo trabajo: no consta. } \\
N=454 \\
\text { Edad = no consta } \\
\text { Gintervención } \\
n=227 \\
\text { Edad }=\text { no consta } \\
\text { Gcontrol } \\
n=227 \\
\text { Edad }=\text { no consta }\end{array}$ & Canadá & 1982-1984 & $\begin{array}{l}\text { Disolventes alifáticos y aromáticos, } \\
\text { plásticos, pesticidas y germicidas, } \\
\text { aceites metales, gas, detergentes, } \\
\text { otros químicos fetotóxicos y no } \\
\text { fetotóxicos }\end{array}$ & $\begin{array}{l}\text { Abortos espontáneos a las } 28 \text { semanas o más en las } \\
\text { trabajadoras expuestas a pesticidas y/o germicidas } \mathrm{OR}=3,1 \\
\text { (IC } 95 \% 1,1-8,6) \text {. } \\
\text { No hubo asociación significativa en el resto de químicos. }\end{array}$ \\
\hline $\begin{array}{l}\text { Ahlborg et al. } \\
\text { 1989(58) }\end{array}$ & Cohortes & $\begin{array}{l}\text { Tipo trabajo: trabajadoras de oficina, } \\
\text { cuidadoras de niños, profesoras y } \\
\text { trabajadoras sociales, enfermeras y } \\
\text { auxiliares de enfermería, fisioterapeutas } \\
\text { y técnicos de laboratorio, comerciales, } \\
\text { limpiadoras, camareras, cocineras, } \\
\text { trabajadoras industriales y agricultoras. } \\
N=3901 \\
\text { Edad = no consta } \\
\text { G. intervención } \\
\text { Sin datos } \\
\text { Gcontrol } \\
\text { Sin datos }\end{array}$ & Suecia & 1980 a 1983 & $\begin{array}{l}\text { Disolventes orgánicos, gases } \\
\text { anestésicos, plomo y otros metales, } \\
\text { otros químicos no especificados }\end{array}$ & $\begin{array}{l}\text { Disolventes orgánicos: } \\
\text { aborto espontáneo/muerte perinatal RR = 0,71 (IC } 95 \% \text { 0,31 } \\
-1,60 \text { ), malformaciones congénitas y bajo peso al nacer RR } \\
=0,57 \text { (IC } 95 \% \text { 0,29- } 1,13 \text { ). Otros químicos: } \\
\text { aborto espontáneo/muerte perinatal RR }=1,27 \text { (IC } 95 \% \text { 0,82 } \\
-1,97) \text {, malformaciones congénitas y bajo peso al nacer RR } \\
=1,28 \text { (IC } 95 \% 0,91-1,80 \text { ). }\end{array}$ \\
\hline $\begin{array}{l}\text { Kyyrönen et al. } \\
\text { 1989(60) }\end{array}$ & Casos y controles & $\begin{array}{l}\text { Tipo trabajo: trabajadoras de lavandería } \\
\text { y de limpieza en seco. } \\
N=536 \\
\text { Edad }=\text { no consta } \\
\text { Gintervención } \\
n=154 \\
\text { Edad }=\text { no consta } \\
\text { Gcontrol } \\
n=382 \\
\text { Edad = no consta }\end{array}$ & Finlandia & 1973-1983 & $\begin{array}{l}\text { Tetracloroetileno, otros disolventes } \\
\text { (sin especificar). }\end{array}$ & $\begin{array}{l}\text { Aborto espontáneo en las trabajadoras expuestas a } \\
\text { tetracloroetileno OR = 3,4 (IC } 95 \% 1,0-11,2 \text { ). } \\
\text { Malformaciones congénitas y exposición a tetracloroetileno } \\
O R=0,8 \text { (IC } 95 \% 0,2-3,5 \text { ) a otros disolventes OR = 5,9 (IC } \\
95 \% 1,0-35,7) \text {. }\end{array}$ \\
\hline
\end{tabular}




\begin{tabular}{|c|c|c|c|c|c|c|}
\hline Primer autor y año & Diseño & Población & País & Periodo & Exposición & Resultado principal \\
\hline $\begin{array}{l}\text { McDonald et al. } \\
1987^{(61)}\end{array}$ & Casos y controles & $\begin{array}{l}\text { Tipo trabajo: manufactura, comercio } \\
\text { mayorista y minorista, hospitales y } \\
\text { servicios y ciertos tipos de } \\
\text { docencia e investigación en artes y } \\
\text { ciencias. } \\
N=602 \\
\text { Edad = no consta } \\
\text { Gintervención } \\
n=174 \\
\text { Edad = no consta } \\
\text { Gcontrol } \\
n=428 \\
\text { Edad = no consta }\end{array}$ & Canadá & 1982 a 1984 & $\begin{array}{l}\text { Disolventes alifáticos, disolventes } \\
\text { aromáticos, plastificantes y plásticos, } \\
\text { metales, aceites, detergentes, gases, } \\
\text { un grupo combinado de bactericidas, } \\
\text { fungicidas y pesticidas, } \\
\text { y un resto diverso }\end{array}$ & $\begin{array}{l}\text { Mayor prevalencia de malformaciones congénitas en } \\
\text { el grupo de casos (63:47). Solo exposición a solventes } \\
\text { aromáticos (especialmente a tolueno) mostró una clara } \\
\text { prevalencia de malformaciones congénitas (18:8; p = 0,04), } \\
\text { más evidente en el } \\
\text { grupo del tracto urinario (9:0). }\end{array}$ \\
\hline $\begin{array}{l}\text { Hemminki et al. } \\
1985^{(62)}\end{array}$ & Casos y controles & $\begin{array}{l}\text { Tipo trabajo: enfermeras. } \\
N=588 \\
\text { Edad = no consta } \\
\text { Gintervención } \\
n=217 \\
\text { Edad = no consta } \\
\text { Gcontrol } \\
n=571 \\
\text { Edad = no consta }\end{array}$ & Finlandia & 1973-1979 & $\begin{array}{l}\text { Gases anestésicos, agentes } \\
\text { esterilizantes (óxido de etileno, } \\
\text { glutaraldehído, formaldehído), } \\
\text { hexaclorofeno, medicamentos } \\
\text { citostáticos }\end{array}$ & 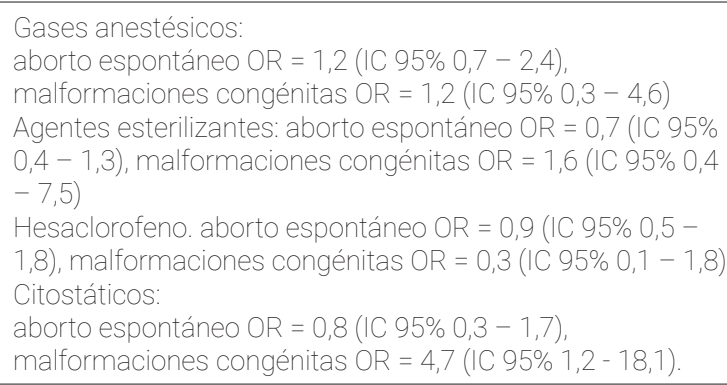 \\
\hline $\begin{array}{l}\text { Axelsson et al. } \\
1984^{(63)}\end{array}$ & Cohortes & $\begin{array}{l}\text { Tipo trabajo: técnicos y asistentes de } \\
\text { laboratorios de facultades de medicina, } \\
\text { odontología y ciencias naturales. } \\
N=745 \\
\text { Edad }=<19 \text { a }>35 \text { años } \\
\text { Gintervención } \\
\text { Sin datos } \\
\text { Gcontrol } \\
\text { Sin datos }\end{array}$ & Suecia & 1968 a 1979 & Disolventes orgánicos & $\begin{array}{l}\text { La tasa de aborto espontáneo RR }=1,31 \text { (IC 95\% 0,89 -1,91). } \\
\text { Sin diferencias en las tasas de mortalidad perinatal o la } \\
\text { prevalencia de malformaciones congénitas. }\end{array}$ \\
\hline $\begin{array}{l}\text { OR = Odds Ratio; RR = Riesgo } \\
\text { Relatico; IC = Intervalo de } \\
\text { confianza }\end{array}$ & & & & & & \\
\hline
\end{tabular}

Tabla 2: Análisis de la calidad documental de los estudios a través de los 22 puntos de valoración de la guía STROBE

Artículo Puntuación de los puntos del cuestionario ${ }^{a}$

\begin{tabular}{|c|c|c|c|c|c|c|c|c|c|c|c|c|c|c|c|c|c|c|c|c|c|c|c|c|}
\hline & 1 & 2 & 3 & 4 & 5 & 6 & 7 & 8 & 9 & 10 & 11 & 12 & 13 & 14 & 15 & 16 & 17 & 18 & 19 & 20 & 21 & 22 & TOTAL & $\%^{b}$ \\
\hline Makelarski et al. (28) & 0,5 & 1 & 1 & 0 & 1 & 1 & 1 & 1 & 0 & NA & 1 & 1 & 0,6 & 1 & 1 & 1 & 0 & 1 & 1 & 1 & 1 & 1 & 17,16 & 81,7 \\
\hline$\underset{(29)}{\text { Vaktskjold et al. }}$ & 1 & 1 & 1 & 0 & 1 & 1 & 1 & 1 & 0 & NA & 1 & 1 & 0,6 & 1 & 1 & 1 & 1 & 1 & 1 & 1 & 0 & 1 & 17,66 & 84,1 \\
\hline $\begin{array}{l}\text { Testud et al. } \\
\text { (30) }\end{array}$ & 0,5 & 1 & 1 & 0 & 1 & 1 & 1 & 1 & 0 & NA & NA & 1 & 0,3 & 0,3 & 1 & 1 & 0 & 1 & 1 & 1 & 1 & 1 & 15,16 & 75,8 \\
\hline
\end{tabular}




\begin{tabular}{|c|c|c|c|c|c|c|c|c|c|c|c|c|c|c|c|c|c|c|c|c|c|c|c|c|}
\hline Garlantézec et al. (31) & 1 & 1 & 1 & 1 & 1 & 0,5 & 1 & 1 & 0 & NA & 1 & 1 & 0,3 & 1 & 1 & 1 & 0 & 1 & 0 & 1 & 1 & 1 & 16,83 & 80,1 \\
\hline Halliday-Bell et al. ${ }^{(32)}$ & 0,5 & 1 & 1 & 0 & 1 & 0,5 & 1 & 1 & 0 & NA & 1 & 1 & 0,3 & 1 & 1 & 1 & 0 & 1 & 1 & 1 & 0 & 0 & 14,3 & 68,1 \\
\hline Vaktskjold et al. (33) & 0,5 & 1 & 1 & 1 & 1 & 1 & 1 & 1 & 0 & NA & 1 & 1 & 0,6 & 1 & 1 & 1 & 0 & 1 & 0 & 1 & 1 & 1 & 17,17 & 81,7 \\
\hline $\begin{array}{l}\text { Shirangi et al. } \\
\text { (34) }\end{array}$ & 1 & 1 & 1 & 0 & 1 & 1 & 1 & 1 & 1 & NA & 1 & 1 & 0,6 & 1 & 1 & 1 & 1 & 1 & 1 & 1 & 1 & 1 & 19,66 & 93,6 \\
\hline $\begin{array}{l}\text { Frey et al. } \\
(35)\end{array}$ & 1 & 1 & 1 & 1 & 1 & 1 & 1 & 1 & 0 & NA & 1 & 1 & 1 & 1 & 1 & 1 & 0 & 1 & 0 & 1 & 0 & 1 & 17,00 & 80,95 \\
\hline $\begin{array}{l}\text { Chevrier et al. } \\
\text { (36) }\end{array}$ & 1 & 1 & 1 & 1 & 1 & 1 & 1 & 1 & 1 & NA & 1 & 1 & 0,6 & 1 & 1 & 1 & 0 & 1 & 1 & 1 & 1 & 1 & 19,66 & 93,6 \\
\hline $\begin{array}{l}\text { Vaktskjold et al. } \\
(37)\end{array}$ & 1 & 1 & 1 & 0 & 1 & 1 & 1 & 1 & 0 & NA & 1 & 1 & 0,6 & 1 & 1 & 1 & 1 & 1 & 1 & 1 & 1 & 1 & 19,66 & 93,6 \\
\hline $\begin{array}{l}\text { Wennborg et al. } \\
\text { (38) }\end{array}$ & 0,5 & 1 & 1 & 1 & 1 & 1 & 1 & 1 & 0 & NA & 1 & 1 & 1 & 1 & 1 & 1 & 0 & 1 & 0 & 1 & 0 & 1 & 16,5 & 78,6 \\
\hline$\underset{\text { (39) }}{\text { Shaw et al. }}$ & 1 & 1 & 1 & 1 & 1 & 1 & 1 & 1 & 0 & NA & 1 & 1 & 0,6 & 0,3 & 1 & 1 & 0 & 1 & 1 & 1 & 0 & 1 & 16,99 & 80,9 \\
\hline $\begin{array}{l}\text { Shaw et al. } \\
(40)\end{array}$ & 0,5 & 1 & 1 & 1 & 1 & 1 & 0,5 & 1 & 0 & NA & 1 & 0,5 & 0,6 & 0 & 1 & 1 & 0 & 1 & 1 & 1 & 0 & 0 & 14,16 & 67,4 \\
\hline $\begin{array}{l}\text { Schüz et al. } \\
(41)\end{array}$ & 0,5 & 1 & 1 & 1 & 1 & 1 & 0,5 & 1 & 0 & NA & 1 & 1 & 0,3 & 1 & 1 & 1 & 0 & 0 & 1 & 1 & 1 & 0 & 15,33 & 73 \\
\hline $\begin{array}{l}\text { Lorente et al. } \\
(42)\end{array}$ & 1 & 1 & 1 & 1 & 1 & 1 & 1 & 1 & 0 & NA & 1 & 1 & 0,6 & 1 & 1 & 1 & 0 & 0,5 & 1 & 1 & 0 & 1 & 17,16 & 81,7 \\
\hline$\underset{(43)}{H}$ Hrubá et al. & 0,5 & 1 & 1 & 0 & 1 & 0,5 & 1 & 1 & 0 & NA & 1 & 1 & 0 & 0 & 1 & 0,3 & 0 & 0 & 0 & 1 & 1 & 1 & 12,33 & 58.7 \\
\hline $\begin{array}{l}\text { Khattak et al. } \\
\text { (44) }\end{array}$ & 1 & 1 & 1 & 0,5 & 1 & 1 & 1 & 1 & 0 & NA & 1 & 1 & 0,6 & 1 & 1 & 1 & 0 & 0 & 1 & 1 & 1 & 1 & 17,16 & 81,7 \\
\hline $\begin{array}{l}\text { Seidler et al. } \\
(45)\end{array}$ & 0,5 & 1 & 1 & 0 & 1 & 1 & 1 & 1 & 0 & NA & 1 & 1 & 0 & 0,3 & 1 & 0,3 & 0 & 1 & 1 & 1 & 1 & 1 & 14,16 & 67,4 \\
\hline $\begin{array}{l}\text { García et al. } \\
(46)\end{array}$ & 1 & 1 & 1 & 1 & 1 & 0,5 & 0,5 & 1 & 0 & NA & 1 & 0 & 0,6 & 0,3 & 1 & 0,3 & 0 & 0 & 0 & 1 & 1 & 0 & 11,32 & 53,9 \\
\hline$\underset{(47)}{\text { Hewitt et al. }}$ & 1 & 1 & 1 & 1 & 1 & 1 & 0,6 & 0 & 0 & NA & 0 & 1 & 0,3 & 0,3 & 1 & 1 & 0 & 0,5 & 1 & 1 & 1 & 0 & 13,82 & 65,8 \\
\hline$\underset{(48)}{\text { Doyle et al. }}$ & 1 & 1 & 1 & 1 & 1 & 0,5 & 0,5 & 0,5 & 0 & NA & 0,5 & 1 & 0,6 & 0,6 & 0,5 & 1 & 0 & 1 & 0 & 1 & 1 & 1 & 14,82 & 70,6 \\
\hline $\begin{array}{l}\text { Agnesi et al. } \\
(49)\end{array}$ & 1 & 1 & 1 & 1 & 1 & 1 & 1 & 0 & 0 & NA & 1 & 1 & 0,6 & 0,4 & 1 & 1 & 0 & 1 & 1 & 1 & 0 & 0 & 15,00 & 71,4 \\
\hline $\begin{array}{l}\text { Laumon et al. } \\
\text { (50) }\end{array}$ & 1 & 1 & 1 & 1 & 1 & 1 & 0,5 & 0 & 0 & NA & 0 & 0 & 0,3 & 0,3 & 0 & 1 & 0,5 & 1 & 0 & 1 & 1 & 0 & 11,66 & 55,5 \\
\hline
\end{tabular}




\begin{tabular}{|c|c|c|c|c|c|c|c|c|c|c|c|c|c|c|c|c|c|c|c|c|c|c|c|c|}
\hline $\begin{array}{l}\text { Swan et al. } \\
\text { (51) }\end{array}$ & 1 & 0,5 & 0,5 & 0 & 1 & 1 & 1 & 1 & 1 & NA & 1 & 1 & 0 & 0 & 1 & 1 & 0 & 0 & 0 & 1 & 1 & 0 & 13,00 & 61,9 \\
\hline $\begin{array}{l}\text { Irwin et al. } \\
(52)\end{array}$ & 0,5 & 1 & 1 & 1 & 1 & 0,5 & 1 & 1 & 0 & NA & 1 & 0 & 0,6 & 0,6 & 1 & 1 & 0 & 1 & 0 & 1 & 1 & 0 & 14,32 & 68,2 \\
\hline $\begin{array}{l}\text { Taskinen et al. } \\
\text { (53) }\end{array}$ & 1 & 1 & 1 & 0 & 1 & 1 & 1 & 1 & 0 & NA & 1 & 1 & 0,3 & 0,3 & 1 & 0,3 & 0 & 1 & 0 & 1 & 1 & 1 & 14,99 & 71,4 \\
\hline${ }_{(54)}^{\text {McAbee et al. }}$ & 0 & 1 & 1 & 1 & 0,5 & 1 & 1 & 1 & 1 & 1 & 1 & 0 & 0,3 & 0,3 & 1 & 1 & 1 & 1 & 1 & 1 & 1 & 1 & 18,16 & 82,54 \\
\hline $\begin{array}{c}\text { Roeleveld et al. } \\
\text { (55) }\end{array}$ & 1 & 1 & 0 & 1 & 1 & 1 & 1 & 1 & 1 & NA & 1 & 0 & 0,3 & 0,6 & 1 & 0,3 & 0 & 1 & 1 & 1 & 1 & 0 & 15,32 & 72,9 \\
\hline $\begin{array}{l}\text { Cordier et al. } \\
(56)\end{array}$ & 1 & 1 & 0 & 1 & 1 & 1 & 1 & 1 & 0 & NA & 1 & 0 & 0,3 & 0,3 & 1 & 1 & 0 & 1 & 0 & 0 & 1 & 1 & 13,66 & 65 \\
\hline 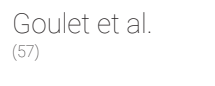 & 0,5 & 1 & 1 & 1 & 1 & 1 & 1 & 1 & 1 & NA & 1 & 1 & 0,3 & 0,3 & 1 & 0 & 1 & 1 & 0 & 1 & 1 & 0 & 16,6 & 79 \\
\hline $\begin{array}{l}\text { Ahlborg et al. } \\
\text { (58) }\end{array}$ & 1 & 1 & 1 & 0 & 1 & 1 & 1 & 1 & 0 & NA & 1 & 1 & 0,3 & 0 & 1 & 0,3 & 0 & 1 & 1 & 1 & 1 & 1 & 15,66 & 74,6 \\
\hline $\begin{array}{l}\text { Kyyrönen et al. } \\
(59)\end{array}$ & 1 & 1 & 1 & 1 & 1 & 1 & 1 & 0,5 & 1 & NA & 0 & 0 & 1 & 0,3 & 1 & 1 & 0 & 1 & 0 & 1 & 1 & 1 & 15,8 & 75,2 \\
\hline $\begin{array}{l}\text { McDonald et al. } \\
\left({ }^{0}\right)\end{array}$ & 1 & 1 & 1 & 1 & 0 & 1 & 1 & 0 & 0 & NA & 0 & 0 & 0 & 0 & 0 & 0 & 0 & 0,5 & 0 & 1 & 0 & 0 & 7,50 & 35,7 \\
\hline$\underset{(61)}{\text { Hemminki et al. }}$ & 0 & 1 & 1 & 0 & 1 & 0,5 & 1 & 0 & 0,5 & NA & 0 & 1 & 0,3 & 0 & 1 & 1 & 0 & 1 & 0 & 1 & 1 & 0 & 11,3 & 53,8 \\
\hline $\begin{array}{l}\text { Axelsson et al. } \\
(62)\end{array}$ & 0 & 1 & 0 & 0 & 1 & 0,5 & 1 & 1 & 0,5 & NA & 1 & 1 & 0,3 & 0 & 1 & 1 & 0 & 1 & 0 & 1 & 1 & 0 & 12,3 & 58,6 \\
\hline
\end{tabular}


Tabla 3: Efectos derivados de la exposición laboral en las mujeres trabajadoras embarazadas expuestas a sustancias peligrosas

Resultado

Alteraciones congénitas

\section{Artículo}

Vaktskjold et al. 2011

Testud et al. 2010 (30)

Garlantézec et al. 2009 (3)

Chevrier et al. $2006^{(3)}$

Vaktskjold et al. 2006

Wennborg et al. $2005^{(38}$

Shaw et al. 2003 (39:

Shaw et al. $2001^{(40)}$

Lorente et al. 2000 (42)

Hrubá et al. 1999 (48

Khattak et al. 1999 (44)

García et al. 1998 (44)

Hewitt et al. 1998 (47)

Laumon et al. 1996 (50)

Taskinen et al. 1994

Cordier et al. $1992^{(566}$

Ahlborg et al. 1989 (58)

Kyyrönen et al. 1989

McDonald et al. 1987 (60

Hemminki et al. $1985^{(67)}$

Axelsson et al. 1984 (62)
Asociación

No

Sí

Sí

(2)

No

Sí

No

So

Sí

Sí

No

Sí

No

No

No

Sí

Sí

No

No
$\mathrm{OR}=0,9($ IC95\% 0,7-1, 1$)$

$\mathrm{OR}=1,24(\mathrm{IC} 95 \% 0,85-1,82)$

$\mathrm{OR}=2,48($ IC $95 \%$ 1,4-4,4)

Ver tabla 1

$\mathrm{OR}=0,85(1 \mathrm{C} 95 \% 0.52-1,26)$

$\mathrm{OR}=1,8(\mathrm{IC} 95 \%$ 1,0 -2,9)

$\mathrm{OR}>1,5$

$\mathrm{OR}<2,0$

Ver tabla 1

$O R=2,31(p<0,05)$

$\mathrm{RR}=13,0(\mathrm{IC} 95 \%$ 1,8-99,5)

$\mathrm{OR}=6,18(\mathrm{IC} 95 \% 1,48-25,69)$

$R R=0,5($ IC 95\% 0,02-3,2)

$O R=0,3($ IC95\% 0,1-0,9)

Ver tabla 1

$R R=1,28(195 \% 0,91-1,80)$.

Exposición

Pesticidas

Disolventes orgánicos

Disolventes orgánicos

Disolventes orgánicos

Níquel

Disolventes orgánicos

Múltiples químicos

Múltiples químicos

Múltiples químicos

Disolventes orgánicos

Disolventes orgánicos

Disolventes orgánicos

Disolventes orgánicos

Disolventes orgánicos

plom

Disolventes orgánicos

Disolventes orgánicos

Gases anestésicos, citostáticos

$\mathrm{OR}=4,7($ IC $95 \%$ 1,2- 18,1)

Disolventes orgánicos 


\begin{tabular}{|c|c|c|c|c|c|}
\hline \multirow{2}{*}{$\begin{array}{l}\text { Resultado } \\
\text { Aborto espontánec }\end{array}$} & & \multirow{2}{*}{$\begin{array}{l}\text { Artículo } \\
\text { Testud et al. } 2010 \text { (30) }\end{array}$} & \multicolumn{2}{|c|}{ Asociación } & \multirow{2}{*}{$\begin{array}{l}\text { Exposición } \\
\text { Disolventes orgánicos }\end{array}$} \\
\hline & & & No & $p>0,05$ & \\
\hline & & Vaktskjold et al. 2008 (33) & No & $O R=1,14(\operatorname{IC~} 95 \% 0,95-1,37)$ & Níquel \\
\hline & & Shirangi et al. $2008^{\left({ }^{(3)}\right)}$ & Si & Ver tabla 1 & Gases anestésicos, pesticidas, radiaciones \\
\hline & & Frey et al. 2007 (35) & No & Sin datos & Disolventes orgánicos \\
\hline & & Doyle et al. 1997 (48) & Sí & $\mathrm{OR}=1,67(\mathrm{IC} 95 \% 1,17-2,36)$ & Disolventes orgánicos \\
\hline & & Agnesi et al. 1997 (49) & Sí & $\mathrm{RR}=3,85(\mathrm{IC} 95 \% 1,24-11,9)$ & Disolventes orgánicos \\
\hline & & Swan et al. 1995 (51) & Sí & $\mathrm{RR}=3,21(\mathrm{IC} 95 \% 1,29-5,96)$ & Disolventes orgánicos \\
\hline & & Taskinen et al. 1994 (53) & Sí & Ver tabla 1 & Disolventes orgánicos \\
\hline & & McAbee et al. $1993^{(54)}$ & Sí & $\mathrm{OR}=3,78(\operatorname{IC~} 95 \% 1,12-12,78)$ & $\begin{array}{l}\text { Disolventes orgánicos, medicamentos } \\
\text { antineoplásicos, radiaciones }\end{array}$ \\
\hline & & Goulet et al. $1991^{(57)}$ & Sí & $O R=3,1(\operatorname{IC~} 95 \% 1,1-8,6)$ & Disolventes orgánicos \\
\hline & & Ahlborg et al. 1989 (58) & No & $R R=0,71($ IC $95 \% 0,31-1,60)$ & $\begin{array}{l}\text { Disolventes orgánicos, gases anestésicos, } \\
\text { metales }\end{array}$ \\
\hline & & Kyyrönen et al. 1989 (59) & Sí & $\mathrm{OR}=3,4(\mathrm{IC} 95 \% 1,0-11,2)$ & Disolventes orgánicos \\
\hline & & Hemminki et al. $19855^{(61)}$ & No & Ver tabla 1 & Gases anestésicos, citostáticos \\
\hline & & Axelsson et al. 1984 (62) & No & $\mathrm{RR}=1,31(\mathrm{IC} 95 \% 0,89-1,91)$ & Disolventes orgánicos \\
\hline \multirow[t]{13}{*}{ Otros resultados } & \multirow[t]{3}{*}{ Parto pretérmino } & Halliday-Bell et al. 2009 (32) & Sí & Ver tabla 1 & Productos de peluquería y estética \\
\hline & & Frey et al. $2007^{(35)}$ & No & Sin datos & Disolventes orgánicos \\
\hline & & Hewitt et al. 1998 (47) & No & $\mathrm{RR}=1,3(\mathrm{IC} 95 \% 0,5-3,3)$ & Disolventes orgánicos \\
\hline & \multirow[t]{3}{*}{ Bajo peso al nacer } & Halliday-Bell et al. 2009 (32) & Sí & Ver tabla 1 & Productos de peluquería y estética \\
\hline & & Hewitt et al. $1998(47)$ & No & $\mathrm{RR}=1,5(\mathrm{IC} 95 \% 0,4-4,7)$ & Disolventes orgánicos \\
\hline & & Ahlborg et al. 1989 (58) & No & $R R=0,71(\operatorname{IC~} 95 \% 0,31-1,60)$ & $\begin{array}{l}\text { Disolventes orgánicos, gases anestésicos, } \\
\text { metales }\end{array}$ \\
\hline & \multirow[t]{4}{*}{ Muerte perinatal } & Halliday-Bell et al. 2009 (32) & Sí & Ver tabla 1 & Productos de peluquería y estética \\
\hline & & Frey et al. 2007 (35) & No & Sin datos & Disolventes orgánicos \\
\hline & & Ahlborg et al. 1989 (58) & No & $R R=0,57($ IC $95 \% 0,29-1,13)$ & $\begin{array}{l}\text { Disolventes orgánicos, gases anestésicos, } \\
\text { metales }\end{array}$ \\
\hline & & Axelsson et al. 1984 (62) & No & Sin datos & Disolventes orgánicos \\
\hline & \multirow{3}{*}{$\begin{array}{l}\text { Hipertensión arterial } \\
\text { inducida por el embarazo }\end{array}$} & Frey et al. $2007^{(35)}$ & No & Sin datos & Disolventes orgánicos \\
\hline & & Hewitt et al. $19988^{(47)}$ & Sí & $\mathrm{RR}=3,5(\mathrm{IC} 95 \% 1,0-12,2)$ & Disolventes orgánicos \\
\hline & & Irwin et al. 1994 (52) & No & $R R=0,68$ & Agentes químicos no especificados \\
\hline
\end{tabular}




\section{Financiación}

Este trabajo no contó con ningún tipo de financiación.

\section{Conflicto de intereses}

Las autoras y los autores de la presente revisión declaran la no existencia de conflicto de interés.

\section{Bibliografía}

1. Instituto Nacional de Seguridad y Salud en el Trabajo (INSST). Directrices para la evaluación de riesgos y protección de la maternidad en el trabajo. Madrid: INSST; 2011. https://bit.ly/2OqXyCm

2. Rodríguez Ortiz de Salazar B, Jardón Dato E, Maqueda Blasco J, Álvarez Sáenz JJ. Riesgos laborales durante el embarazo. Med Segur Trab. 2005;51:53-63.

3. Directiva 92/85/CEE del Consejo. Comunicación de la Comisión sobre las directrices para la evaluación de los agentes químicos, físicos y biológicos, así como los procedimientos industriales considerados como peligrosos para la salud o la seguridad de la trabajadora embarazada, que haya dado a luz o en período de lactancia. COM/2000/0466 final, de 5 de noviembre de 2000. https://bit.ly/30vpxaE

4. Ley 39/1999, de 5 de noviembre, para promover la conciliación de la vida familiar y laboral de las personas trabajadoras. Boletín Oficial del Estado 266, de 6 de noviembre de 1999. https://www.boe.es/eli/es///1999/11/05/39/con

5. Real Decreto Legislativo 1/1994, de 20 de junio, por el que se aprueba el texto refundido de la Ley General de la Seguridad Social. Boletín Oficial del Estado 154, de 29 de junio de 1994. https://www.boe.es/eli/es/rdlg/1994/06/20/1/con

6. Ley 31/1995, de 8 de noviembre, de prevención de Riesgos Laborales. Boletín Oficial del Estado 269, de 10 de noviembre de 1995. https://www.boe.es/eli/ es/l/1995/11/08/31

7. Rodríguez Ortiz de Salazar B, Martínez Herrera JA, Álvarez Collado L, Silva Mato A. Prestación por riesgos laborales durante el embarazo en Madrid 20002004. Med Segur Trab. 2006;52:15-28.

8. Villar R, Benavides FG, Serra L, Serra C. Prestación por riesgo durante el embarazo e incapacidad temporal en una cohorte de trabajadoras del Parc de Salut Mar (Barcelona, España). Gac Sanit. 2019;33:455-61.

9. Real Decreto 298/2009, de 6 de marzo, por el que se modifica el Real Decreto 39/1997, de 17 de enero, por el que se aprueba el Reglamento de los Servicios de Prevención, en relación con la aplicación de medidas para promover la mejora de la seguridad y de la salud en el trabajo de la trabajadora embarazada, que haya dado a luz o en período de lactancia. Boletín Oficial del Estado 57, de 7 de marzo de 2009. https://www.boe.es/eli/es/rd/2009/03/06/298 
10. Sejbaek CS, Pedersen J, Schlünssen V, Begtrup LM, Juhl M, Bonde JP, et al. The influence of multiple occupational exposures on absence from work in pregnancy: a prospective cohort study. Scand J Work Environ Health. 2020;46:60-8. doi:10.5271/ sjweh.38408119.

11. Henrotin J-B, Béringuier $H$, Groupe de Travail de L'étude GaT-Hospi G de T de LG-H. Travailler à I'hôpital durant la grossesse: une étude descriptive nationale rétrospective en France. Sante Publique. 2020;31:611-21. doi:10.3917/ spub. 195.0611

12. Antolínez Ruiz P, Lafaurie Villamil MM. Estrés y sobrecarga laboral durante el embarazo: experiencias de médicas del sector asistencial en Bogotá. Hacia Promoc Salud. 2017;22:84-100. doi:10.17151/hpsal.2017.22.1.7

13. Frey G, Schuster M, Oberlinner C, Queier-Wahrendorf A, Lang S, Yong M. Pregnant Employee Protection Program in a Large Chemical Company. J Occup Environ Med. 2015;57:965-72. doi:10.1097/JOM.0000000000000505

14. Bidstrup SB, Kaerlev L, Thulstrup AM, Bonde JPE. Women referred for occupational risk assessment in pregnancy have no increased risk of adverse obstetric outcomes. Dan Med J. 2015;62:A5119.

15. Nugteren JJ, Snijder CA, Hofman A, Jaddoe VWV, Steegers EAP, Burdorf A. Work-related maternal risk factors and the risk of pregnancy induced hypertension and preeclampsia during pregnancy. The Generation R Study. PLoS One. 2012;7:e39263. doi:10.1371/journal.pone.0039263

16. Larsen AD. The effect of maternal exposure to psychosocial job strain on pregnancy outcomes and child development. Dan Med J. 2015;62:B5015.

17. Langlois PH, Hoyt AT, Desrosiers TA, Lupo PJ, Lawson CC, Waters MA, et al. Maternal occupational exposure to polycyclic aromatic hydrocarbons and small for gestational age offspring. Occup Environ Med. 2014;71:529-35. doi:10.1136/ oemed-2013-101833

18. Mayhoub F, Berton $T$, Bach $V$, Tack K, Deguines $C$, Floch-Barneaud $A$, et al. Self-reported parental exposure to pesticide during pregnancy and birth outcomes: the MecoExpo cohort study. PLoS One. 2014;9:e99090. doi:10.1371/journal. pone.0099090

19. Lojewski J, Flothow A, Harth V, Mache S. Employed and expecting in Germany: A qualitative investigation into pregnancy-related occupational stress and coping behavior. Work. 2018;59:183-99. doi:10.3233/WOR-172673

20. Zachek CM, Schwartz JM, Glasser M, DeMicco E, Woodruff TJ. A screening questionnaire for occupational and hobby exposures during pregnancy. Occup Med (Lond). 2019;69:428-35. doi:10.1093/occmed/kqz094

21. Ghatan CE, Fassiotto M, Jacobsen JP, Sze DY, Kothary N. Occupational Radiation Exposure during Pregnancy: A Survey of Attitudes and Practices among Interventional Radiologists. J Vasc Interv Radiol. 2016;27:1013-1020.e3. doi:10.1016/j. jvir.2016.03.040 
22. Jahnke SA, Poston WSC, Jitnarin N, Haddock CK. Maternal and Child Health Among Female Firefighters in the U.S. Matern Child Health J. 2018;22:922-31. doi:10.1007/s10995-018-2468-3

23. Henrotin J-B, Vaissière M, Etaix M, Dziurla M, Malard S, Lafon D. Exposition aux risques professionnels pendant la grossesse : retour de services médicaux interentreprises. Gynecol Obstet Fertil Senol. 2018;46:20-7. doi:10.1016/j. gofs.2017.10.029

24. Wanden-Berghe C, Sanz-Valero J. Systematic reviews in nutrition: standardized methodology. Br J Nutr. 2012;107:S3-7. doi:10.1017/S0007114512001432

25. von Elm E, Altman DG, Egger M, Pocock SJ, Gøtzsche PC, Vandenbroucke JP, et al. The Strengthening the Reporting of Observational Studies in Epidemiology (STROBE) statement: guidelines for reporting observational studies. Gac Sanit. 2008;22:144-50. Doi:10.1157/13119325

26. Harbour R, Miller J. A new system for grading recommendations in evidence based guidelines. BMJ. 2001;323:334-36. doi:10.1136/bmj.323.7308.334

27. Ley 14/2007, de 3 de julio, de Investigación biomédica. Boletín Oficial del Estado 159, de 4 de julio de 2007. https://www.boe.es/eli/es///2007/07/03/14

28. Makelarski JA, Romitti PA, Rocheleau CM, Burns TL, Stewart PA, Waters MA, et al. Maternal periconceptional occupational pesticide exposure and neural tube defects. Birth Defects Res A Clin Mol Teratol. 2014;100:877-86. doi:10.1002/ bdra.23293

29. Vaktskjold A, Talykova LV, Nieboer E. Congenital anomalies in newborns to women employed in jobs with frequent exposure to organic solvents--a register-based prospective study. BMC Pregnancy Childbirth. 2011;11:83. doi:10.1186/14712393-11-83

30. Testud F, D'Amico A, Lambert-Chhum R, Garayt C, Descotes J. Pregnancy outcome after risk assessment of occupational exposure to organic solvents: a prospective cohort study. Reprod Toxicol. 2010;30:409-13. doi:10.1016/j.reprotox.2010.06.004

31. Garlantézec R, Monfort C, Rouget F, Cordier S. Maternal occupational exposure to solvents and congenital malformations: a prospective study in the general population. Occup Environ Med. 2009;66:456-63. doi:10.1136/oem.2008.041772

32. Halliday-Bell JA, Gissler M, Jaakkola JJK. Work as a hairdresser and cosmetologist and adverse pregnancy outcomes. Occup Med (Lond). 2009;59:180-84. doi:10.1093/occmed/kqp017

33. Vaktskjold A, Talykova LV, Chashchin VP, Odland JØ, Nieboer E. Spontaneous abortions among nickel-exposed female refinery workers. Int J Environ Health Res. 2008;18:99-115. doi:10.1080/09603120701498295 
34. Shirangi A, Fritschi L, Holman CDJ. Maternal occupational exposures and risk of spontaneous abortion in veterinary practice. Occup Environ Med. 2008;65:71925. doi:10.1136/oem.2007.035246

35. Frey GM, Ott MG, Messerer P, Nasterlack M, Queisser-Luft A, Zober A. Pregnancy protection program in a large chemical company: design and initial survey results. J Occup Environ Med. 2007;49:507-18. doi:10.1097/JOM.0b013e31805c7454

36. Chevrier C, Dananché B, Bahuau M, Nelva A, Herman C, Francannet C, et al. Occupational exposure to organic solvent mixtures during pregnancy and the risk of non-syndromic oral clefts. Occup Environ Med. 2006;63:617-23. doi:10.1136/ oem.2005.024067

37. Vaktskjold A, Talykova LV, Chashchin VP, Nieboer E, Thomassen Y, Odland JO. Genital malformations in newborns of female nickel-refinery workers. Scand J Work Environ Health. 2006;32:41-50. doi:10.5271/sjweh.975

38. Wennborg H, Magnusson LL, Bonde JP, Olsen J. Congenital malformations related to maternal exposure to specific agents in biomedical research laboratories. J Occup Environ Med. 2005;47:11-9. doi:10.1097/01.jom.0000150237.67801.93

39. Shaw GM, Nelson V, lovannisci DM, Finnell RH, Lammer EJ. Maternal occupational chemical exposures and biotransformation genotypes as risk factors for selected congenital anomalies. Am J Epidemiol. 2003;157:475-84. doi:10.1093/ aje/kwg013

40. Shaw GM, Selvin S, Carmichael SL, Schaffer DM, Nelson V, Neri E. Assessing combined chemical exposures as risk factors for neural tube defects. Reprod Toxicol. 2001;15:631-35. doi:10.1016/s0890-6238(01)00180-0

41. Schüz J, Kaletsch U, Meinert R, Kaatsch P, Michaelis J. Risk of childhood leukemia and parental self-reported occupational exposure to chemicals, dusts, and fumes: results from pooled analyses of German population-based case-control studies. Cancer Epidemiol Biomarkers Prev. 2000;9:835-38.

42. Lorente $C$, Cordier $S$, Bergeret $A$, De Walle HE, Goujard J, Aymé S, et al. Maternal occupational risk factors for oral clefts. Occupational Exposure and Congenital Malformation Working Group. Scand J Work Environ Health. 2000;26:137-45. doi:10.5271/sjweh.523

43. Hrubá D, Kukla L, Tyrlík M. Occupational risks for human reproduction: ELSPAC Study. European Longitudinal Study of Pregnancy and Childhood. Cent Eur J Public Health. 1999;7:210-15.

44. Khattak S, K-Moghtader G, McMartin K, Barrera M, Kennedy D, Koren G. Pregnancy outcome following gestational exposure to organic solvents: a prospective controlled study. JAMA. 1999;281:1106-09. doi:10.1001/jama.281.12.1106

45. Seidler A, Raum E, Arabin B, Hellenbrand W, Walter U, Schwartz FW. Maternal occupational exposure to chemical substances and the risk of infants smaIl-for-gestational-age. Am J Ind Med. 1999;36:213-22. doi:10.1002/(sici)1097-027 4(199907)36:1<213::aid-ajim30>3.0.co;2-a 
46. García AM, Fletcher T. Maternal occupation in the leather industry and selected congenital malformations. Occup Environ Med. 1998;55:284-86. doi:10.1136/ oem.55.4.284

47. Hewitt JB, Tellier L. Risk of adverse outcomes in pregnant women exposed to solvents. J Obstet Gynecol Neonatal Nurs. 1998;27:521-31. doi:10.1111/j.1552-6909.1998.tb02618.x

48. Doyle P, Roman E, Beral V, Brookes M. Spontaneous abortion in dry cleaning workers potentially exposed to perchloroethylene. Occup Environ Med. 1997;54:84853. doi:10.1136/oem.54.12.848

49. Agnesi R, Valentini F, Mastrangelo G. Risk of spontaneous abortion and maternal exposure to organic solvents in the shoe industry. Int Arch Occup Environ Health. 1997;69:311-16. doi:10.1007/s004200050153

50. Laumon B, Martin JL, Collet P, Bertucat I, Verney MP, Robert E. Exposure to organic solvents during pregnancy and oral clefts: a case-control study. Reprod Toxicol. 1996;10:15-9. doi:10.1016/0890-6238(95)02013-6

51. Swan SH, Beaumont JJ, Hammond SK, VonBehren J, Green RS, Hallock MF, et al. Historical cohort study of spontaneous abortion among fabrication workers in the Semiconductor Health Study: agent-level analysis. Am J Ind Med. 1995;28:75169. doi:10.1002/ajim.4700280610

52. Irwin DE, Savitz DA, St André KA, Hertz-Picciotto I. Study of occupational risk factors for pregnancy-induced hypertension among active duty enlisted Navy personnel. Am J Ind Med. 1994;25:349-59. doi:10.1002/ajim.4700250305

53. Taskinen $H$, Kyyrönen P, Hemminki K, Hoikkala M, Lajunen K, Lindbohm ML. Laboratory work and pregnancy outcome. J Occup Med. 1994;36:311-19. doi:10.1097/00043764-199403000-00008

54. McAbee RR, Gallucci BJ, Checkoway H. Adverse reproductive outcomes and occupational exposures among nurses: an investigation of multiple hazardous exposures. AAOHN J. 1993;41:110-9. doi:10.1177/216507999304100301

55. Roeleveld N, Zielhuis GA, Gabreëls F. Mental retardation and parental occupation: a study on the applicability of job exposure matrices. $\mathrm{Br} \mathrm{J}$ Ind Med. 1993;50:945-54. doi:10.1136/oem.50.10.945

56. Cordier S, Ha MC, Ayme S, Goujard J. Maternal occupational exposure and congenital malformations. Scand J Work Environ Health. 1992;18:11-7. doi:10.5271/ sjweh. 1613

57. Goulet L, Thériault G. Stillbirth and chemical exposure of pregnant workers. Scand J Work Environ Health. 1991;17:25-31. doi:10.5271/sjweh.1738

58. Ahlborg G, Hogstedt C, Bodin L, Bárány S. Pregnancy outcome among working women. Scand J Work Environ Health. 1989;15:227-33. doi:10.5271/sjweh.1858

59. Kyyrönen $P$, Taskinen $H$, Lindbohm ML, Hemminki K, Heinonen OP. Spontaneous abortions and congenital malformations among women exposed to tetra- 
chloroethylene in dry cleaning. J Epidemiol Community Health. 1989;43:346-51. doi:10.1136/jech.43.4.346

60. McDonald JC, Lavoie J, Côté R, McDonald AD. Chemical exposures at work in early pregnancy and congenital defect: a case-referent study. $\mathrm{Br} \mathrm{J}$ Ind Med. 1987;44:527-33. doi:10.1136/oem.44.8.527

61. Hemminki K, Kyyrönen P, Lindbohm ML. Spontaneous abortions and malformations in the offspring of nurses exposed to anaesthetic gases, cytostatic drugs, and other potential hazards in hospitals, based on registered information of outcome. J Epidemiol Community Health. 1985;39:141-7. doi:10.1136/jech.39.2.141

62. Axelsson G, Lütz C, Rylander R. Exposure to solvents and outcome of pregnancy in university laboratory employees. Br J Ind Med. 1984;41:305-12. doi:10.1136/ oem.41.3.305

63. Troncoso-Piñeiro P, González de Giarratana AE, Rivadulla-Lema I, Torres-Romero MG, Sanz-Valero J. Neoplasias en trabajadores expuestos al aluminio y/o sus compuestos: Revisión sistemática. Med Segur Trab. 2018;64:312-26.

64. Álvarez Velásquez S, Sanz Valero J. Ventajas de la quimioterapia domiciliaria en los enfermos adultos con neoplasias: revisión sistemática. Hosp Domic. 2020;4:25-41. doi:10.22585/hospdomic.v4i1.98

65. Muñoz-Cobo-Orosa B, Varela-Serrano C, Rodriguez-Ledott M, Sanz-Valero J. Lesiones malignas de la piel en trabajadores del sector pesquero: revisión sistemática. Arch Prev Riesgos Labor. 2021;24:47-61. doi:10.12961/aprl.2021.24.01.05

66. Glasziou P, Vandenbroucke JP, Chalmers I. Assessing the quality of research. BMJ. 2004;328:39-41. doi:10.1136/bmj.328.7430.39

67. Manterola C, Asenjo-Lobos C, Otzen T. Jerarquización de la evidencia: Niveles de evidencia y grados de recomendación de uso actual. Rev Chil Infectol. 2014;31:705-18. doi:10.4067/S0716-10182014000600011

68. Correa A, Stewart WF, Yeh HC, Santos-Burgoa C. Exposure measurement in case-control studies: reported methods and recommendations. Epidemiol Rev. 1994;16:18-32. doi:10.1093/oxfordjournals.epirev.a036142

69. Vandenbroucke JP, von Elm E, Altman DG, Gøtzsche PC, Mulrow CD, Pocock SJ, et al. Strengthening the Reporting of Observational Studies in Epidemiology (STROBE): explanation and elaboration. Int J Surg. 2014;12:1500-24. doi:10.1016/j. ijsu.2014.07.014

70. Melián-Fleitas L, Franco-Pérez ÁM, Sanz-Valero J. Análisis bibliométrico y temático de la producción científica sobre salud laboral relacionada con nutrición, alimentación y dieta, indexada en MEDLINE. Med Segur Trab. 2019;65:10-23.

71. Hernández Hernández H, González Perfetti AE, Torres Romero MG, Rivadulla Lema I, Troncoso Piñeiro P, Sanz-Valero J. Características de las trabajadoras embarazadas y procedimientos de protección de la maternidad: estudio multicéntrico. Rev Asoc Esp Med Trab. 2020;29:340-50. 
72. Domingo-Pueyo A, Sanz-Valero J, Wanden-Berghe C. Efectos sobre la salud de la exposición laboral al cromo y sus compuestos: revisión sistemática. Arch Prev Riesgos Labor. 2014;17:142-53. doi:10.12961/aprl.2014.17.3.03

73. Mancheño Potenciano MC, Izquierdo García MÁ, editores. Exposición laboral a disolventes. Madrid: Unión Sindical de Madrid Región de CCOO; 2008. https://bit. ly/3jYhmMb

74. Ballester Arias AR, García AM. Asociación entre la exposición laboral a factores psicosociales y la existencia de trastornos musculoesqueléticos en personal de enfermería: revisión sistemática y meta-análisis. Rev Esp Salud Publica. 2017;91:e201704028.

75. Calera Rubio AA, Roel Valdés JM, Casal Lareo A, Gadea Merino R, Rodrigo Cencillo F. Riesgo químico laboral: elementos para un diagnóstico en España. Rev Esp Salud Publica. 2005;79:283-95. doi:10.1590/S1135-57272005000200014

76. Vargas Marcos F. Prevención y control del riesgo de los productos químicos. Rev Esp Salud Publica. 1996;70:409-20.

77. Saavedra-Ontiveros D, Arteaga-Martínez M, Serrano-Medina B, Reynoso-Arizmendi F, Prada-Garay N, Cornejo-Roldán LR. Contaminación industrial con solventes orgánicos como causa de teratogénesis. Salud Publica Mex. 1996;38:3-12.

78. Cordier S, Garlantézec R, Labat L, Rouget F, Monfort C, Bonvallot N, et al. Exposure during pregnancy to glycol ethers and chlorinated solvents and the risk of congenital malformations. Epidemiology. 2012;23:806-12. doi:10.1097/EDE. 0b013e31826c2bd8

79. National Institute for Occupational Safety and Health (NIOSH). The effects of workplace hazards on female reproductive health. Cincinnati, USA: NIOSH; 1999. https://bit.ly/2P3qvb8

80. Pak VM, Powers M, Liu J. Occupational chemical exposures among cosmetologists: risk of reproductive disorders. Workplace Health Saf. 2013; 61:5228;quiz529. doi:10.1177/216507991306101204

81. Delvigne A, Vandromme J. Assessment of environmental knowledge and needs among assisted reproductive technology professionals. J Assist Reprod Genet. 2020;37:2347-55. doi:10.1007/s10815-020-01888-2

82. Kumar S, Sharma A, Kshetrimayum C. Environmental and occupational exposure and female reproductive dysfunction. Indian J Med Res. 2019;150:532-45. doi:10.4103/ijmr.ijmr_1652_17

83. Snijder CA., te Velde E, Roeleveld N, Burdorf A. Occupational exposure to chemical substances and time to pregnancy: a systematic review. Hum Reprod Updat. 2012;18:284-300. doi:10.1093/humupd/dms005

84. Bernabeu-Martínez MA, Ramos Merino M, Santos Gago JM, Álvarez Sabucedo LM, Wanden-Berghe C, Sanz-Valero J. Guidelines for safe handling of hazardous 
drugs: A systematic review. PLoS One. 2018;13:e0197172. doi:10.1371/journal. pone. 0197172

85. Hagger MS. What makes a 'good' review article? Some reflections and recommendations. Health Psychol Rev. 2012;6:141-46. doi:10.1080/17437199.2012.70 5556 